\title{
Mitochondrial Metabolism in Myocardial Remodeling and Mechanical Unloading: Implications for Ischemic Heart Disease
}

\author{
Min Jiang ${ }^{1,2,3}$, Xiaoye Xie ${ }^{1,3,4}$, Feng Cao ${ }^{1,3 *}$ and Yabin Wang ${ }^{1,3 *}$ \\ ${ }^{1}$ Department of Cardiology, National Clinical Research Center for Geriatric Disease, The Second Medical Center, Chinese \\ People's Liberation Army General Hospital, Beijing, China, ${ }^{2}$ College of Pulmonary and Critical Care Medicine, Chinese \\ People's Liberation Army General Hospital, Beijing, China, ${ }^{3}$ Medical School of Chinese People's Liberation Army, Chinese \\ People's Liberation Army General Hospital, Beijing, China, ${ }^{4}$ Department of Cadre Ward, The 960 Hospital of Chinese \\ People's Liberation Army, Jinan, China
}

\section{OPEN ACCESS}

Edited by:

Shijun Wang

Fudan University, China

Reviewed by:

Xiaoqiang Tang,

Sichuan University, China

Rui Zhang,

Tongji University, China

*Correspondence:

Yabin Wang

wyb8202@163.com

Feng Cao

fengcao8828@163.com

Specialty section:

This article was submitted to Cardiovascular Metabolism

a section of the journal

Frontiers in Cardiovascular Medicine

Received: 04 October 2021 Accepted: 04 November 2021 Published: 09 December 2021

Citation: Jiang M, Xie X, Cao F and Wang Y (2021) Mitochondrial Metabolism in Myocardial Remodeling and Mechanical Unloading: Implications for Ischemic Heart Disease. Front. Cardiovasc. Med. 8:789267.

doi: 10.3389/fcvm.2021.789267
Ischemic heart disease refers to myocardial degeneration, necrosis, and fibrosis caused by coronary artery disease. It can lead to severe left ventricular dysfunction (LVEF $\leq 35-40 \%)$ and is a major cause of heart failure (HF). In each contraction, myocardium is subjected to a variety of mechanical forces, such as stretch, afterload, and shear stress, and these mechanical stresses are clinically associated with myocardial remodeling and, eventually, cardiac outcomes. Mitochondria produce $90 \%$ of ATP in the heart and participate in metabolic pathways that regulate the balance of glucose and fatty acid oxidative phosphorylation. However, altered energetics and metabolic reprogramming are proved to aggravate HF development and progression by disturbing substrate utilization. This review briefly summarizes the current insights into the adaptations of cardiomyocytes to mechanical stimuli and underlying mechanisms in ischemic heart disease, with focusing on mitochondrial metabolism. We also discuss how mechanical circulatory support (MCS) alters myocardial energy metabolism and affects the detrimental metabolic adaptations of the dysfunctional myocardium.

Keywords: ischemic heart disease, heart failure, mitochondrial metabolism, veno-arterial ECMO, mechanical unloading, left ventricular assist device

\section{INTRODUCTION}

As estimated by World Health Organization (WHO), ischemic heart disease (IHD) is the leading cause of death worldwide (1). IHD stands for disease syndromes caused by myocardial ischemia due to disproportionate supply and demand of oxygenated blood perfusion in the heart (2). The insufficient oxygen, decreased nutrient levels, as well as deficient substrates and metabolites, are all pathophysiological contributors for IHD. Myocardial ischemia is usually induced by diminished coronary blood flow due to atherosclerosis-induced coronary artery occlusion, clinically manifesting as coronary heart disease or coronary artery disease.

IHD affects cardiac metabolism and function in different stages, ranging from angina to acute myocardial infarction (AMI) and HF. In myocardium, mitochondria produce $90 \%$ of ATP, making mitochondrial oxidative phosphorylation (OXPHOS) the primary energy source for the heart. Besides, mitochondria also participate in metabolic pathways that regulate the balance of glucose and fatty acid oxidative phosphorylation. Under physiological conditions, mitochondria keep a 
dynamic and homeostatic state in regulating metabolic pathways and cellular ions, which lay foundation for normal cellular functioning. Any deviation from this homeostatic state will lead to mitochondrial dysfunction, including elevated mitochondrial $\mathrm{Ca}^{2+}$, damage of mitochondria membranes and enzymes and defects of electron transport chain (ETC), all of which are contributing factors for cellular apoptosis and necrosis (3).

In terms of interventions, options for IHD mainly focus on reestablishing the oxygen supply-demand imbalance, which is pivotal for clinical prognosis for patients with IHD. Recently, although related approaches have advanced by leaps and bounds, there are still certain numbers of patients who are refractory to conventional treatment, and some of them develop heart failure (HF) after IHD interventions. As heart is a metabolically active organ, metabolism modulation is promising in the treatment of IHD as energy metabolism is closely related with disease progression.

\section{MITOCHONDRIAL METABOLISM IN NORMAL HEART}

As a circulatory pump, heart requires cardiomyocytes to generate enormous quantities of ATP to sustain the uninterrupted and orderly coordinated systolic and diastolic function. The main fuels of the myocardium are fatty acids (FAs), glucose, pyruvate, lactate, ketone, and amino acids, which are acquired from the blood continuously for the cardiomyocytes cannot store these energy substrates. As previously reported, the majority of energy (60-90\%) required for myocardial activity is derived from free FAs, and the remaining $10-40 \%$ originates from carbohydrates and amino acids (4). The myocardial energy metabolism mainly includes substrate utilization, oxidative phosphorylation, and ATP transfer and utilization. Firstly, acetyl-CoA derived from $\beta$-oxidation of FAs and glycolysis is synthesized in mitochondria and enters the tricarboxylic acid (TCA) cycle. Under various physiological processes, the healthy heart is metabolically flexible in shifting between substrates to maintain energy production. Then, oxidative phosphorylation is completed in the mitochondria through the respiratory chain and generates the direct energy source ATP. Finally, through the creatine phosphate energy shuttle, creatine kinase (CK) in the mitochondria catalyzes ATP and creatine and generates phosphocreatine (PCr) and adenosine diphosphate (ADP). The generated $\mathrm{PCr}$ immediately transfers from mitochondria to myofibrils, where it interacts with ADP and synthesizes ATP and creatine under the catalyzation of myocardial CK so that cardiomyocytes can use ATP for contraction and relaxation and maintain the energy-consuming activity of the heart.

\section{MITOCHONDRIAL METABOLISM IN IHD}

The energy metabolism dysfunction caused by IHD includes alterations of substrate utilization, dysfunction of OXPHOS, impairment of ATP synthesis, and mitochondrial dysfunction. Under physiological conditions, fatty acid oxidation (FAO) generates more ATP than glucose oxidation per molecule oxidized at the expense of greater $\mathrm{O}_{2}$ consumption. In IHD and $\mathrm{HF}$, the $\mathrm{O}_{2}$ supply to the myocardium is insufficient due to reduced tissue perfusion. Accordingly, both FAO and carbohydrate oxidation decline, and production of ATP is disrupted. To compensate the reduced $\mathrm{O}_{2}$ shortage, the ATP production in the myocardium switches from aerobic to anaerobic mode, and glycolysis becomes a more predominant source of energy as it yields $11-13 \%$ more ATP per unit of consumed $\mathrm{O}_{2}$ than $\beta$-oxidation (5). Besides, under ischemic and hypoxic conditions, large quantities of activated longchain FAs produced by fat mobilization accumulate in cells and mitochondrial membranes due to decreased or stopped $\beta$-oxidation of FAs. These long-chain FAs can result in cardiomyocyte damage and death, and suppress the activity of pyruvate dehydrogenase ( $\mathrm{PDH})$, thereby limiting the aerobic oxidation of glucose, reducing the ATP production efficiency, increasing the content of intracellular lactate and hydrogen ions, and, finally, disrupting the myocardial contractility $(3,6)$.

\section{Oxygen Consumption}

It has been increasingly apparent that ischemia and reperfusion (IR)-induced alterations in the kinetics of $\mathrm{O}_{2}$ supply-to-demand equilibrium depend seriously on the duration and severity of IR. The effects of ischemia exist from short, beneficial ischemia $(<10 \mathrm{~min})$ to moderate, reversible ischemia $(10-20 \mathrm{~min})$ to severe, irreversible ischemia (>20 min). In reversible ischemia, recovery of overall oxidative metabolism recovers much slower to a preischemic level and couples to contractile function (7). However, in irreversible ischemia, the recovery of oxygen consumption and metabolism is fast, and the mechanical function is severely damaged (8). The IR-induced increases in $\mathrm{Ca}^{2+}$ partially explain the rapid recovery of metabolism at early reperfusion in irreversible ischemia, whereas the increased glycolytic rate from exogenous glucose induced by IR results in slower recovery of $\mathrm{O}_{2}$ consumption-to-demand balance at late reperfusion (9). The concentration of free $\mathrm{Ca}^{2+}$ is implicated in regulating several intramitochondrial enzymes, including the $\mathrm{PDH}$, the isocitrate dehydrogenase, and the oxoglutarate dehydrogenase, thus leading to elevated carbohydrate oxidation during early reperfusion (10). This supports the notion that the mitochondrion is the first organelle to display signs of injury under irreversible ischemia, and that regulating mitochondrial energetics within the cell is an early protective strategy of reversible ischemia (9). Moreover, the rapid metabolic recovery uncoupled from mechanical function is a surrogate for poor prognosis of IR injury. Therefore, interventions aiming at attenuating reperfusion blood flow or inhibiting mitochondrial function are considered beneficial $(11,12)$. In the reperfusion stage following ischemia, FAO generates ATP as a primary energy source at the cost of glucose oxidation negatively impacts the cardiac function in the isolated hearts and in patients with coronary artery disease $(6,13)$.

\section{Glucose Metabolism}

Glucose metabolism mainly includes anaerobic glycolysis, aerobic oxidation, pentose phosphate pathway (PPP), uronic acid pathway, glycogen synthesis and decomposition, 
gluconeogenesis, and hexose metabolism, etc. As mentioned above, under hypoxia and ischemia, the aerobic oxidation of FAs is diminished and glycolysis increases and glucose becomes the main energy substrate for myocardial energy metabolism. The uptake of glucose depends on glucose transporters (GLUTs) on sarcolemma of cardiomyocytes, and GLUT1 and GLUT4 are two major isoforms in cardiomyocytes. GLUT1 dominates during fetal and early postnatal life, whereas GLUT4 is the main isoform in adult cardiomyocytes. GLUT4 has a higher affinity for glucose than GLUT1, which makes GLUT4 functioning as the major glucose transporter in adult heart (14). Upon ischemia and hypoxia, GLUT4 translocates to cell membrane from the intracellular vesicles and then augments glucose intake (Figure 1A), exhibiting a protective role during cardiac ischemia in neonatal or adult mouse (15-17). Besides, GLUT4 depletion represses glucose use during ischemia and facilitates progression of irreversible cardiac dysfunction associated with blunted ATP generation during IR injury (18). After being transported into cytosol, glucose is phosphorylated by hexokinase, generating glucose 6-phosphate (G6P). Then, G6P is converted into pyruvate by glycolytic enzymes, including phosphofructokinase 1 (PFK1), PFK2, and pyruvate kinase (PK), and the pyruvate can either be metabolized to lactate or be transported into the mitochondrial matrix through the mitochondrial pyruvate carrier (MPC) for oxidation. Under ischemia, the accumulation of 5'-AMP leads to activation of AMP-activated protein kinase (AMPK), which phosphorylates and activates phosphofructokinase-2 (PFK-2) to promote glycolysis $(19,20)$. Recently, elevated MPC1 and MPC2 have been found in the surviving tissue of the heart subjected to IR injury, indicating a compensatory mechanism that enhances mitochondrial pyruvate uptake to limit intracellular acidosis (21). Upon transportation into mitochondria, pyruvate is converted to acetyl-CoA by pyruvate dehydrogenase (PDH) and further metabolized in the TCA cycle. During ischemia, glucose oxidation is completely shuttled down (Figure 1A), and the recovery of it is regulated by FA oxidation as FA competes with glucose for oxidation.

During myocardial reperfusion, the level of $\mathrm{O}_{2}$ and substrates are restored, whereas glycolysis still remains high, because lactate and inorganic phosphoric acid are washed away which diminish the inhibition of glycolysis, and dysfunctional mitochondria cannot use $\mathrm{O}_{2}$ effectively. However, glucose oxidation is inhibited during the early stage of reperfusion. As mentioned above, AMPK is activated during ischemia and the accumulated AMPK inactivates ACC at the early stage of reperfusion (8). The decreased ACC activity, in combination with malonylCoA degradation leads to decreased malonyl-CoA levels during reperfusion, relieving inhibition of CPT1 and, finally, resulting in upregulated FA oxidation $(22,23)$. Increased FA oxidation generates high levels of acetyl-CoA and NADH/NAD ${ }^{+}$, which inhibits the enzyme activity of $\mathrm{PDH}$ through activating PDK4 and therefore inhibits pyruvate oxidation (8). The excessive fatty acids $\beta$-oxidation facilitates the accumulation of intermediates from the TCA cycle and efflux of citrate to cytosol via the mitochondrial citrate carrier (CIC)/SLC25A1, and high levels of cytosolic citrate can suppress $\mathrm{PFK}-1 / 2$ and pyruvate kinase (PK) activities, and thus diminish glycolysis, pyruvate generation, and oxidation $(24,25)$. However, as demonstrated before, the $\mathrm{Ca}^{2+}$ overload during reperfusion may conquer this inhibition by activating $\mathrm{PDH}$ and then increasing glucose oxidation (10, 26). Above all, glucose oxidation is inhibited at an early stage of reperfusion after moderate ischemia, while may increase following more severe or prolonged ischemia $(27,28)$.

Data from in vivo and ex vivo studies suggested that promoting glucose oxidation during perfusion is closely correlated with reduced IR injury and improved cardiac function. In ex vivo studies, interventions increasing glucose oxidation target the $\mathrm{PDH}$ flux, including boosting $\mathrm{PDH}$ activity with dichloroacetate, supplementing pyruvate or facilitating glucose uptake by applying high glucose/insulin or overexpressing GLUT1 transporter (29-31). Simultaneously, repressing FAs uptake or FAO also relieves inhibition of glucose oxidation (32, 33). As the improved function observed in the short reperfusion period mostly indicates the accelerated recovery of mechanical function and enhanced re-energization, the long-term postischemic myocardial salvage is to be assessed. Therefore, in vivo studies administrating glucose oxidation-stimulating substances, such as dichloroacetate, phosphonate compounds, or highdensity lipoproteins (HDL) prior to reperfusion reduced infarct size several days after reperfusion (34-36).

Although increased glycolysis only marginally increases ATP production, it promotes flux into the metabolic pathways branching from glycolysis, such as polyol and hexosamine biosynthetic pathways that could independently activate signaling pathways responsible for myocardial remodeling (37, 38). Besides, it has been suggested that glycolysis is intrinsically linked to the redox state of cell (39). Glucose metabolized through glycolysis and the branching PPP in the cytosol feeds the TCA cycle in the mitochondria and produces major reductant e.g., NADH, NADPH for thioldependent antioxidant defense. Activation of PPP results in upregulated NADPH/NADP ${ }^{+}$and GSH/GSSG couples and an improved redox state, which relieved acute IR injury in cultured cardiomyocytes and mouse hearts (40).

\section{Fatty Acid Oxidation}

After uptake into cardiomyocytes, FAs are esterified to fatty acyl-CoA in cytosol and then transferred to carnitines by carnitine palmitoyl transferase 1 (CPT1), which localizes in the inner surface of mitochondrial outer membrane (41). The acylcarnitines are transferred into mitochondrial matrix by carnitine acylcarnitine translocase (CAT) and then converted back into fatty acyl-CoA by CPT2. The fatty acyl-CoA is catalyzed to acetyl-CoA for $\beta$-oxidation in mitochondria.

Fatty acids metabolism dysfunction includes FAs and carnitine transport disorder, CoA dehydrogenase deficiency, impairment of enzymes, ketones production disorder, etc. CPT-1 is the ratelimiting enzyme for $\beta$-oxidation and is inhibited by MalonylCoA, which is synthesized by acetyl-CoA carboxylase (ACC) from acetyl-CoA using biotin and ATP as cofactors. The level of malonyl-CoA in the heart is regulated both by its synthesis and degradation, and AMPK, ACC, and malonylCoA decarboxylase (MCD) are extremely important. AMPK phosphorylates and inhibits ACC, which reduces the production 


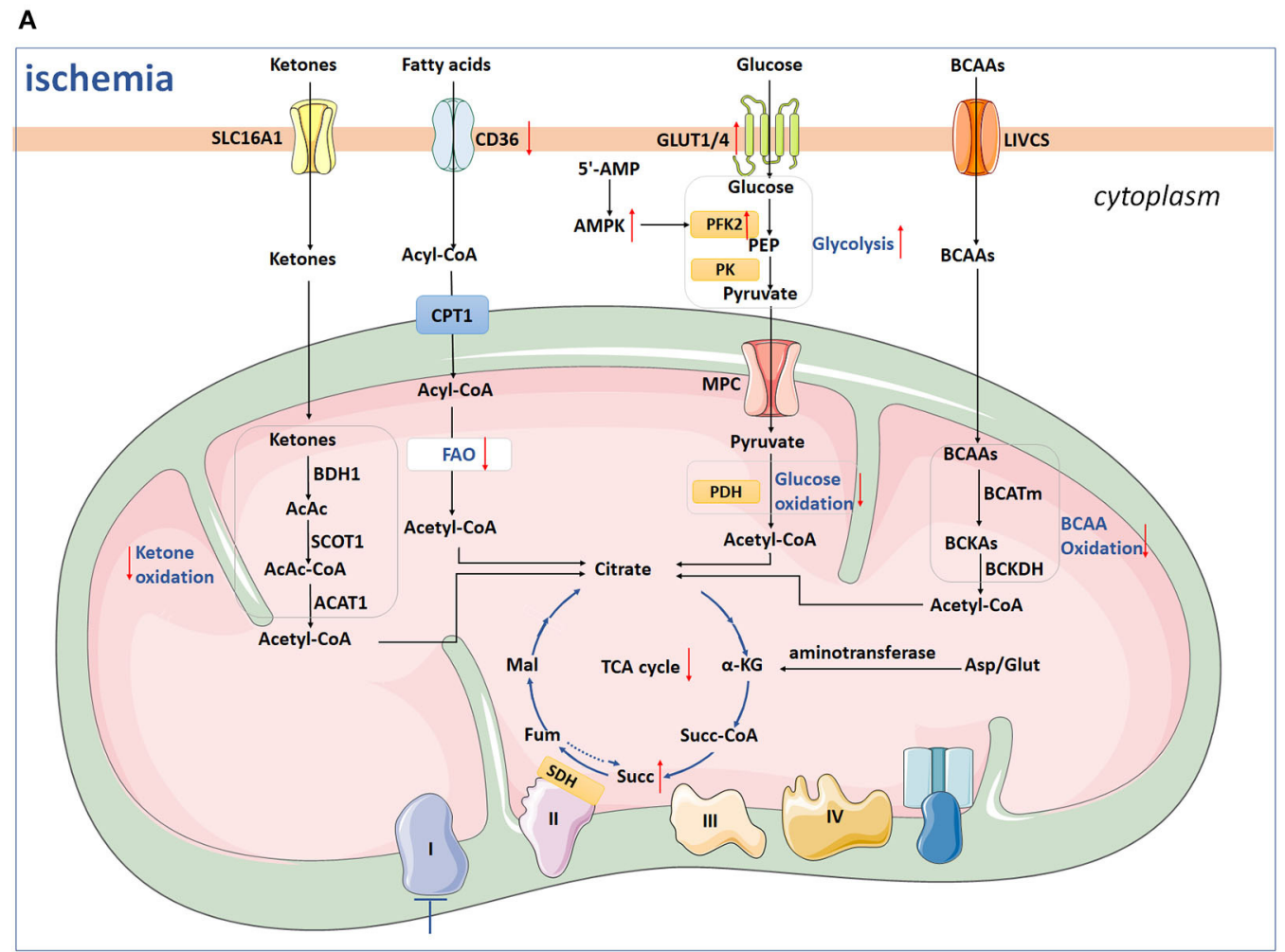

B

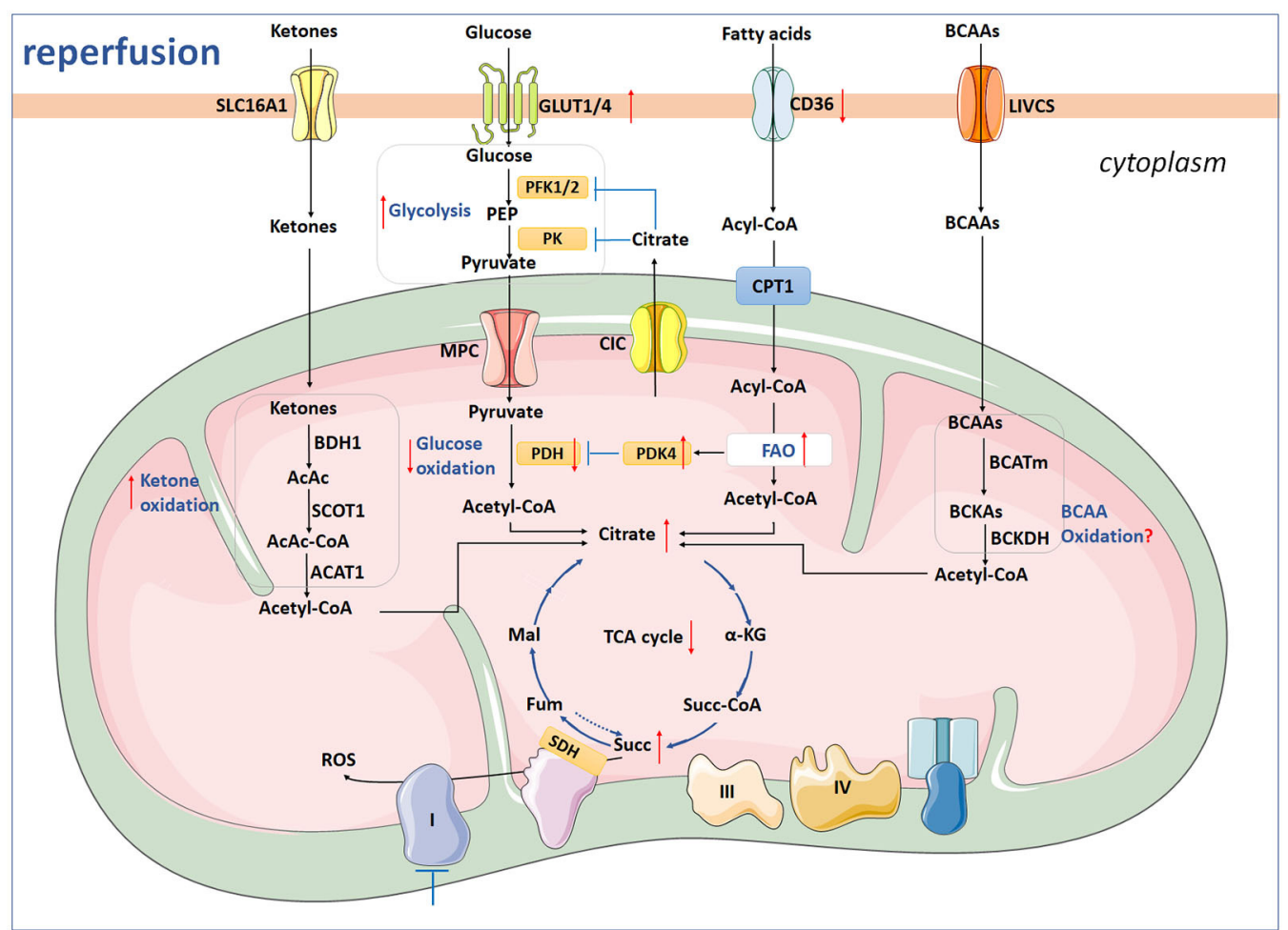

FIGURE 1 | The scheme depicts ketone oxidation, glycolysis, glucose oxidation, fatty acid oxidation, BCAA oxidation, and succinate metabolism under ischemia (A) and reperfusion (B). (A) Under ischemia, GLUT4-mediated glucose uptake and glycolysis are increased by AMPK-induced PFK-2 activation, whereas glucose 
FIGURE 1 | oxidation is shuttled down. The CD36 on sarcolemma, FAs uptake, and FAO are inhibited. Besides, BCAAs and ketones oxidation are also inhibited during ischemia. The TCA cycle and Complex I are inhibited, while the succinate is accumulated from existing metabolites of TCA with mitochondrial Complex II reversal and aminotransferase anaplerosis. (B) During reperfusion, CD36 remains low, while FAO returns to the pre-ischemic level. High levels of NADH, acetyl-CoA, and ATP in mitochondria generated from increased FAO inhibit activated PDH via activating PDK4 and, therefore, inhibit glycolysis and pyruvate oxidation. The glycolysis still remains high, while glucose oxidation is inhibited. Ketones utilization is increased, while the level of BCAAs oxidation remains to be clarified in the future. Two-thirds of succinate enters into perfusate, and the remaining one-third of succinate is oxidized via SDH, driving ROS burst. SLC16A1, Solute carrier (SLC) 16A1/monocarboxylate transporter 1 (MCT1); $\beta$-OHB, $\beta$-hydroxybutyrate; AcAc, acetoacetate; BDH1, $\beta$-hydroxybutyrate dehydrogenase 1; SCOT, succinyl-CoA:3 oxoacid-CoA transferase; ACAC-CoA, catalyze acetoacetyl-CoA; ACAT1, acetyl-CoA acetyltransferase 1; CPT1, carnitine palmitoyl transferase 1; FAO, fatty acids oxidation; GLUT1/4, glucose transporter 1/4; PEP, phosphoenolpyruvate; PK, pyruvate kinase; MPC, mitochondrial pyruvate carrier; PDH, pyruvate dehydrogenase; BCAAs, branched chain amino acids; LIVCS, Leu, lle, Val: cation symporter; BCKAs, $\alpha$-keto-acids; BCATm, mitochondrial branched chain aminotransaminase; $\mathrm{BCKDH}$, branched chain $\alpha$-keto acid dehydrogenase; $\alpha-\mathrm{KG}$, $\alpha$-ketoglutarate; Fum, fumarate; Mal, malate; Succ, succinate; Succ-CoA, succinyl-CoA; SDH, succinate dehydrogenase; Asp, aspartate; Glut, glutamine. The arrow facing up represents an increase, and down indicates a decrease. Blue lines with a T shape represent inhibition.

of malonyl-CoA and phosphorylates as well as activates MCD, promoting the reverse reaction and converting malonyl-CoA into acetyl-CoA (42). As demonstrated before, AMPK was rapidly activated as an energy sensor to regulate FFAs uptake and oxidation under pathophysiological conditions of hypoxia and ischemia. In cardiomyocytes, AMPK promotes long-chainFAs uptake through FA translocase (CD36) (43). AMPK activation and relative increase of MCD decrease myocardial malonyl CoA content, which then alleviates inhibition of CPT1 and subsequently facilitates FAO as increased fatty acyl CoA moieties enters into the mitochondrial matrix (44). During mild ischemia, this facilitates fatty acid $\beta$-oxidation to be the primary and continuous contributor for oxidative ATP generation, which leads to a decreased level of glucose oxidation, despite increased glycolysis (45). Besides, lactate production by $\mathrm{LDH}$ from pyruvate and an increased level of $\mathrm{NAD}^{+}$ from NADH are also promoted in order to sustain anaerobic glycolysis. The uncoupling of glycolysis and glucose oxidation leads to an accumulation of lactate and $\mathrm{H}^{+}$, aggravating the ionic disturbance induced by ischemia. However, it should be noted that the accumulated lactate and $\mathrm{H}^{+}$are immediately eliminated from the cardiomyocyte under conditions of hypoxia and mild ischemia.

With total ischemia, the FAO is inhibited due to the accumulated reducing equivalents and disturbance of acyl CoA dehydrogenase and 3-hydroxyacyl CoA dehydrogenase enzyme reactions (Figure 1A). CD36 on sarcolemma is downregulated by $32 \%$, and the FAO rate decreases by $95 \%$ (46). The inhibition of fatty acid $\beta$-oxidation inevitably leads to accumulation of metabolic intermediates in various cell compartments. Fatty acyl carnitines accumulate in cytosol and the mitochondrial matrix, while fatty acyl-CoA mainly deposits in mitochondrial matrix because the CoA pool does not exchange with the small cytoplasmic CoA pool (47). The excessive amounts of acylcarnitine accelerate ROS production and increase the probability of mitochondrial permeability transition pore (mPTP) opening, and excessive cytosolic acyl-CoA causes loss of $\Delta \Psi \mathrm{m}$ from outside of mitochondria $(47,48)$. This inhibits the formation of amorphous intramitochondrial densities and disrupts mitochondrial cristae, both of which may eventually induce mitochondrial dysfunction (49). Therefore, the approaches aiming at regulating FA metabolism for cardioprotection in IR injury should first evaluate the potential impact on overall energy homeostasis and possible cardiotoxicity carefully. Recently, studies have shown that inhibition of FAO reduces damage induced by IR using inhibitors, which limit FA transport and metabolism pathways $(32,50-54)$.

In the stage of perfusion, the activation of AMPK and increased MCD activity still persist, leading to marked decrease of malonyl CoA content. Therefore, the rates of FAO rapidly return to its pre-ischemic level during reperfusion at the expense of glucose oxidation with the CD36 level remained low (Figure 1B) (46), whereas the myocardial contraction is still depressed. However, this causes the uncoupling of glucose metabolism and thus deteriorates intracellular acidosis, which increases the risk of cardiac arrhythmias and decreases the contractility because the response between contractile proteins and $\mathrm{Ca}^{2+}$ is repressed (55). Therefore, the myocardial recovery is hindered despite the restoration of coronary perfusion.

\section{Ketone Body Oxidation}

Ketone bodies [acetoacetate, acetone, and $\beta$-hydroxybutyrate $(\beta-\mathrm{OHB})]$ are synthesized in the liver using acetyl $\mathrm{CoA}$ that predominately derived from FAO. They are increasingly being recognized as an important energy substrate of the myocardium, especially in $\mathrm{HF}(38,56)$. After entering mitochondria, they rapidly form acetyl-CoA via $\beta$-OHB dehydrogenase (BDH1), succinyl-CoA:3-oxoacid-CoA transferase (SCOT) and mitochondrial acetyl-CoA acetyltransferase 1 (ACAT1) (38). The predominant ketone body oxidized in heart is $\beta$-OHB. In terms of $\mathrm{O}_{2}$ consumption for ATP generation, ketones are much more efficient than FAs but less efficient than glucose (4).

Increasing ketone oxidation rates in failing hearts increases overall energy production without compromising glucose or fatty acid metabolism, albeit without increasing cardiac efficiency (57). Previous studies showed that short-term fasting reduces infarct size and limits the duration of ventricular tachycardia occurring at early reperfusion, and this is correlated with increased concentration of $\beta-\mathrm{OHB}$ and $\beta-\mathrm{OHB} /$ acetoacetate ratio (58). However, whether ketonemia induced by fasting in mice after IR injury protect or deteriorates myocardial injury is conflicting $(59,60)$. Furthermore, in vivo studies suggested that the effect of ketonemia may be boosted by exogenous ketone therapy: fasting with ketone infusion led to the least myocardial damage compared with fasting without ketone infusion or a fed state with ketone infusion in rat hearts after coronary occlusion (61). 
As $\beta$-OHB conferred substantial protection against oxidative stress in multiple organs $(62,63)$, further mechanism study also confirmed that reduced mitochondrial ROS, enhanced ATP generation, attenuated mitochondrial swelling, and partly restored $\mathrm{mPTP}$ in myocardium account for the cardio protection against IR injury (64). To determine ketones utilization under ischemic condition in humans, the concentrations of coronary and systemic serum lactate and $\beta$-OHB were measured in 171 consecutive patients with angina (65). The results showed that increased lactate production was associated with decreased myocardial $\beta$-OHB consumption, demonstrating suppressed utilization of ketone bodies under ischemia in the coronary circulation (Figure 1A) (65).

$\mathrm{HF}$ is the next stage of myocardial infarction. A mouse model of progressive $\mathrm{HF}$ induced by transverse aortic constriction (TAC)/myocardial infarction exhibited upregulated BDH1 along with upregulated genes encoding ketone body transporters (MCT1 and MCT2) and increased 13C-BHB use on the basis of nuclear magnetic resonance studies (66). Cardiac-specific BDH1 knockout mice exhibit more severe ventricular remodeling and dysfunction after TAC/myocardial infarction (66), whereas $\mathrm{BDH} 1$ overexpression attenuates cardiac remodeling and DNA damage in a pressure-overloaded model (67). Increased availability of $3-\mathrm{OHB}$ significantly reduced ventricular remodeling and improved mitochondrial energetics caused by TAC/myocardial infarction in wild-type mice compared with mice-fed normal chow (68). Except for pathway manipulation and ketone supplementation, the cardioprotective effects of SGLT2 inhibitors on infarcted hearts can also be attributed to increased myocardial ketone utilization $(69,70)$.

\section{BCAA Metabolism}

Branched chain amino acids oxidation is also an origin of ATP generation by the heart (71). In mitochondria, leucine, isoleucine, and valine are firstly transaminated to branched chain $\alpha$-keto-acids (BCKAs) by the mitochondrial branched chain aminotransaminase (BCATm), and then decarboxylated to form $\mathrm{CoA}$ compounds by the mitochondrial branched chain $\alpha$-keto acid dehydrogenase (BCKDH). The products of $\mathrm{BCKDH}$ will then generate acetyl-CoA, which fuels for the TCA cycle.

High levels of circulating BCAA and its derivative BCKAs are correlated with increased incidence of cardiovascular disease $(72,73)$. Previous studies revealed that plasma and cardiac levels of BCAA and BCKAs increase in the rodent model of myocardial IR injury $(74,75)$. Impaired cardiac BCAA oxidation exacerbates cardiac insulin resistance and directly contributes to cardiac dysfunction and remodeling post AMI $(74,76)$, while BCAA supplementation aggravates cardiac dysfunction and increases infarct size following coronary artery ligationinduced myocardial infarction (74). As BCAA oxidation rates in heart only supply $1-2 \%$ of the total cardiac ATP (75), it is impossible that interventions increasing BCAA catabolism itself have substantial impact on cardiac ATP generation. Recently, BCAA and their intermediates have been recognized to affect glucose and FA metabolism (76-78). In the heart, chronic accumulation of BCAA inhibits $\mathrm{PDH}$ activity through suppressing protein $\mathrm{O}$-linked-N-acetylglucosamine (O-GlcNAc) modification, leading to significantly reduced glucose oxidation, and, therefore, rendering the heart vulnerable to IR injury (76). Dietary or genetic factors-induced BCAA accumulation enhances cardiac FAO levels via upregulating PPAR- $\alpha$ expression transcriptionally, thereby triggering lipid peroxidation toxicity and rendering the heart vulnerable to IR injury (78). As BCKAs have been shown to trigger ROS formation (79), promoting oxidative decarboxylation of BCKAs through PPC2m overexpression reduces the oxidative stress and eventually alleviates myocardial IR injury (80). However, in contrast with this, another study found that BCKAs attenuate acute IR injury in hearts by inhibiting oxidative stress-induced cell death and protecting mitochondria and energy production (81). The discrepancy may be attributed to the manner of BCKAs exposure and the severity of injury/stress. Moreover, it has been proved that blockade of mitochondria ETC at different complexes inhibits cardiac IR injury $(23,82)$. BCKAs repress activity of complex I in cardiac mitochondria (83), which capacitate its function as mitochondrial targets for cardioprotection.

\section{Succinate Metabolism}

When exposed to ischemia, succinate, a metabolite of the TCA cycle, accumulates and is oxidized by succinate dehydrogenase (SDH) which drives enormous ROS production by mitochondrial complex I (84). Previous study by Zhang and colleagues found that succinate mainly originates from TCA cycle using existing metabolites, such as fumarate, malate, oxaloacetate, citrate, and aconitate, with only trivial contributions from mitochondrial complex II reversal and aminotransferase anaplerosis during heart ischemia (Figure 1A) (85). Glucose derived from glycogen also seems to play a part in ischemia induced-succinate accumulation without the requirement of mitochondrial pyruvate entry, suggesting that glucose is not the main source of carbohydrates (85). The $\alpha$-ketoglutarate $(\alpha-K G)$-derived succinate is protective at the first few minutes of ischemia as it contains GTP generation through phosphorylating succinyl-CoA synthetase at the substrate level, which defers ischemia-induced ATP exhaustion in cardiomyocytes (86).

Not all succinate that accumulates during ischemia is oxidized by SDH, with approximately two-thirds of them release into the perfusate via MCT1 under low $\mathrm{pH}$ conditions during early reperfusion (87). The remaining one-third succinate are quickly oxidized within a few minutes of reperfusion, which diminishes the CoQ pool and also hyperpolarizes the inner membrane of mitochondria (84). Together, these alterations drive reverse electron transport (RET) at complex I, which produces ROS dramatically (Figure 1B) $(84,88)$. Besides, succinate oxidation induces generation and secretion of pro-inflammatory factors, such as IL-1 $\beta$, and simultaneously inhibits production of antiinflammatory factors (89).

Clinically, the succinate concentration in arterial, coronary sinus, and peripheral venous blood of patients with STEMI is higher than that in patients with non-STEMI or stable angina, and the release of cardiac succinate in STEMI is correlated with the severity of acute myocardial injury (90). The emerging therapeutic and cardioprotective strategy targeting 
succinate metabolism via $\mathrm{SDH}$ inhibition includes preventing its accumulation during ischemia (less oxidizable succinate during reperfusion) and directly depressing its oxidation during reperfusion (91). The TCA cycle intermediate malate and the succinate competitor malonate are known SDH inhibitors (86). $\mathrm{SDH}$ inhibition with malonate before ischemia or at the onset of reperfusion reduces infarct size in isolated mice hearts through inhibiting ROS generation and MPTP opening (84, 92). The discovery that one third of accumulated succinate during ischemia is oxidized within the first $5 \mathrm{~min}$ of reperfusion (and $59 \%$ of which is within the 1 st $\mathrm{min}$ ) suggests that early reperfusion is a critical time window for therapeutic targeting of complex II (85). Furthermore, SDH inhibition by malonate administration at 1-week post-MI facilitates adult cardiomyocyte proliferation, revascularization of the infarct zone, and myocardial regeneration following infarction (93). Above all, succinate represents a promising biomarker for evaluating severity of AMI, and inhibition of SDH proposes a key metabolic target to render protection following infarction.

\section{Epigenetic Aspects of Metabolic Signaling}

Most of the previously described substrate utilization and the metabolic process are a result of gene transcription. During the last decades, epigenetics, including DNA modification and posttranslational modification of proteins, are increasingly studied to delineate how metabolites regulate gene expression. Lysine acetylation, one of the posttranslational modifications, can regulate many non-histone proteins, which are involved in gene transcription, mRNA splicing, signal transduction, cell metabolism and survival (94). Sirtuins (SIRT1-7) are Class III histone deacetylases (HDACs), which participate in IR injury via regulating mitochondrial metabolism. In the heart mitochondria of SIRT5 knockout mice, mass spectrometry suggests that lysine succinylation is increased and the substrate of SIRT5 includes proteins that participate in FA metabolism, TCA cycle and OXPHOS (95). Compared with wild-type littermates, SIRT5 knockout exacerbates infarction, and this is reversed by pretreatment of malonate, implicating SIRT5 modulates protein succinylation in IR injury. SIRT3 is the primary mitochondrial lysine deacetylase; its knockdown significantly aggravates the extent of IR injury compared to wild-type hearts (96). The enhanced mitochondrial protein acetylation, which is closely correlated with the inhibited activity of complex I and MnSOD, is the underlying mechanism. In the sepsis model, depletion of SIRT3 also induces excessive acetylation of enzymes in the TCA cycle and production of lactate and $\mathrm{NADH}$, which accordingly deteriorate sepsis-induced cardiac dysfunction (97). However, recently, a dual knockout mouse model targeting carnitine acetyltransferase and SIRT3 resulted in a dramatically proteomewide upregulation of lysine acetylation in heart mitochondria, which exhibited a $\sim 86 \%$ overlap with characteristics of HF, while this has no or minimal impact on mitochondrial function, such as dehydrogenase activity, respiration, redox state and OXPHOS (98). Therefore, clinical and mechanistic studies are to be done to determine protein acetylation in mitochondrial metabolism and its actual contribution to IR injury.
Except for acetylation and succinylation, new acylation mediated by metabolites themselves is founded to participate in epigenetic regulation of gene expression and enzymatic activity. In an HFpEF (HF with preserved ejection fraction) mice model, by partly activating citrate synthase through lysine $\beta$ hydroxybutyrylation and inhibiting FA uptake, $\beta$-OHB limits the acetyl-CoA pool and thus inhibits mitochondrial acetylation (99). Besides, the crotonylation of histone also regulates gene transcription and metabolism. In hypertrophic cardiomyopathy, Enoyl Coenzyme A Hydratase1 (ECHS1), the enzyme in the second step of fatty acids $\beta$-oxidation, reduces the intracellular crotonyl-CoA level and inhibits cardiomyocytes hypertrophy via repressing $\mathrm{H} 3 \mathrm{~K} 18 \mathrm{cr}$ and $\mathrm{H} 2 \mathrm{BK} 12 \mathrm{cr}$ and NFATc3 expression (100). Recently, Zhang et al. have discovered that lactate, the product of glycolysis, can stimulate histone lactylation, which participates in M1 macrophage polarization by inducing Arg1 expression (101). The histone lactylation is later found to regulate tissue repair and cancer progression (101-103). As macrophage phenotype and inflammation are closely correlated with prognosis of IHD and HF $(104,105)$, the role of this "lactate clock" in cardiovascular system is to be clarified to develop novel therapeutics.

\section{MECHANICAL CIRCULATORY SUPPORT ON MYOCARDIAL MITOCHONDRIAL METABOLISM}

In spite of timely perfusion, over $1 / 4$ of patients suffering the firsttime AMI develop HF within 1 year (106). For every 5\% increase in the myocardial infarct size, the 1-year HF hospitalization and 1-year mortality will increase by $20 \%$ (107). The reason for the poor prognosis of patients with AMI is that reperfusion paradoxically worsens myocardial injury. Upon reperfusion, the ischemic myocardium is subjected to abrupt biochemical and metabolic alterations, including mitochondrial re-energization, ROS burst, intracellular and mitochondrial $\mathrm{Ca}^{2+}$ overload, rapid $\mathrm{pH}$ correction and inflammation (108). These alterations can lead necrosis of endothelial cells and cardiomyocytes via opening of $\mathrm{MPTP}$ and hypercontraction. The MPTP is a nonselective channel on inner membrane of mitochondria, and its opening disrupts the mitochondrial membrane potential, which uncouples OXPHOS, exhausts ATP, and induces cell death. During ischemia, mPTP is closed, while the reperfusioninduced ROS burst, $\mathrm{Ca}^{2+}$ overload, and rapid $\mathrm{pH}$ fluctuation cause mPTP opening (109). Therefore, mitochondria, acting as a signaling hub, are an important target for cardioprotection. Improved and more efficient strategies are in urgent demand to limit myocardial injury by inhibiting deleterious response and inducing a cardioprotective mechanism prior to reperfusion of the infarct-related arteries so as to prevent the development of patients with HF after MI. Recently, increasing numbers of mechanical circulatory support (MCS) devices have been applied in clinical practice as they can enhance systemic mean arterial pressure and concurrently reduce left ventricular (LV) wall stress and stroke work, which may be beneficial for myocardial oxygen supply-demand equilibrium (110-112). 


\section{Mechanical Loading on Mitochondrial Metabolism of Myocardium}

Normally, cardiomyocytes are subjected to three types of mechanical loading, including cyclic stretch imposed by every heartbeat, shear stress generated by flowing blood, and static stretching due to blood pressure. Cardiomyocytes are sensitive to mechanical stress, which are transduced to molecular signaling by biomechanical sensors. The sensors and transduction pathways have been studied for several years in the hope of preventing development and progression of cardiovascular diseases by shutting down mechanotransduction.

Emerging evidence has shown that excessive mechanical loading can disturb mitochondrial homeostasis, including dynamics, metabolism, $\mathrm{Ca}^{2+}$ homeostasis and redox state (113, 114). $\mathrm{Ca}^{2+}$ leak is the earliest alteration observed under mechanical loading, and during myocardial systole, the duration and amount of $\mathrm{Ca}^{2+}$ leak determine the contractile force of myocardium (115). $\mathrm{Ca}^{2+}$ is a versatile signaling molecule, which regulates numerous cellular processes, including cell metabolism, and the mitochondrial $\mathrm{Ca}^{2+}$ homeostasis is determine by equilibrium between $\mathrm{Ca}^{2+}$ influx and efflux, which are regulated by $\mathrm{Ca}^{2+}$ channels, transporters, exchangers and subcellular organelles $(116,117) . \mathrm{Ca}^{2+}$ affects metabolic processes such as the TCA cycle through allosteric regulation of enzymes or indirectly via regulating phosphatases and kinases $(118,119)$. In addition, $\mathrm{Ca}^{2+}$ channels, transporters and exchangers are also reported to be responsible for metabolism (120-122). The $\mathrm{Ca}^{2+}$ releaseactivated $\mathrm{Ca}^{2+}$ (CRAC) channel, which imports $\mathrm{Ca}^{2+}$ from endoplasmic reticulum and extracellular space, is involved in FA metabolism (119). Piezo1, which is discovered in 2010 by Coste et al. and forms cationic non-specific channels, also participates in mechanotransduction by transducing ionic current and $\mathrm{Ca}^{2+}$ influx (123). In cardiovascular system, Piezol expresses in endothelial cells, smooth muscle cells, cardiomyocytes, and cardiac fibroblasts and can be activated by cyclic stretch and shear stress. In endothelial cells, Piezol couples shear stressprovoked $\mathrm{Ca}^{2+}$ entry and endothelial cell organization and alignment via $\mathrm{Ca}^{2+}$-activated proteolytic enzyme calpain (124). However, data on the presence and function of Piezol in cardiomyocytes are limited. Liang et al. found that the mRNA and the protein level of Piezol were upregulated in HF after myocardial infarction (125). In another in vitro model, Piezo1 was reported to participate in sensing cyclic stretch and can activate the mechanical low-density lipoprotein (LDL) receptorrelated protein 6 (LRP6)/ $\beta$-catenin signaling pathway (126). Clinically, the increased Piezol mRNA level was observed on monocytes of patients with aortic valve stenosis, which increases shear stress on circulating blood cells, and transcatheter aortic valve implantation (TAVI) significantly decreased the mRNA level on monocytes (127). Further in vitro mechanistic study suggests that Piezol-mediated $\mathrm{Ca}^{2+}$ influx from intracellular stores, as well as the extracellular environment, was involved in monocyte activation. Apart from $\mathrm{Ca}^{2+}$ homeostasis, Piezol constitutes a regulator of the Hippo-Yes-associated protein (YAP)/PDZ-binding motif (TAZ) pathway $(128,129)$, which is involved in converting mechanical stress into biochemical signal and has been well-studied (130). With regular mechanical stress, the activated Hippo proteins phosphorylate YAP/TAZ and thus confine them to cytoplasm. Upon excessive mechanical loading, Hippo is inhibited and YAP/TAZ enters into nucleus where it interacts with the TEAD1 family to induce gene transcription. YAP and TAZ can regulate metabolism of various substrates and mitochondrial function, which has been reviewed in detail previously (131). However, the direct evidence that Piezol regulates mitochondrial metabolism is lacking.

\section{Unloading by LV-To-Aorta Circulatory Support}

Over the past decades, preclinical studies have demonstrated that, compared with reperfusion alone, mechanical unloading of the left ventricle prior to coronary reperfusion can reduce IR injury and myocardial infarct size in AMI $(132,133)$. Latterly, preclinical studies also indicated that LV unloading and delayed reperfusion by $30 \mathrm{~min}$ can initiate cardioprotective changes, including upregulated SDF-1a/CXCR4 expression, increased cardioprotective signaling, and reduced apoptosis $(133,134)$. Compared with reperfusion alone, $30 \mathrm{~min}$ of LV mechanical unloading before reperfusion was adequate to diminish LV infarct size, improve cardiac function, and downregulate biomarkers related to undesirable remodeling and HF 28 days post AMI, thus promoting recovery of myocardial function 30 days after AMI (135). Furthermore, mechanical unloading increased collateral coronary flow and myocardial microvascular perfusion by reducing the LV-end diastolic wall stress (136, 137). In order to determine the safety and feasibility of LV unloading and delayed reperfusion of patients with STEMI, the DTU-STEMI pilot trial (Door-To-Unload in STEMI Pilot Trial) randomly assigns 50 patients with anterior STEMI to LV unloading with immediate reperfusion or LV unloading followed by 30-min delay before reperfusion (138). The results of the trial suggested that, despite delayed reperfusion, LV unloading does not increase the infarct size, which lays a good foundation for a future pivotal trial. In addition, in patients with large anterior STEMIs, which is defined as a sum of precordial STsegment elevation STE $\geq 6 \mathrm{~mm}$, LV unloading reduces infarct size normalized to the area at risk compared with reperfusion alone (138). In patients with LAD occlusion and STE $\geq 7 \mathrm{~mm}, \mathrm{LV}$ unloading also diminishes the infarct size quantified by cardiac magnetic resonance at 3- to 5-day post-MI compared with that in the perfusion-alone group (139).

\section{Unloading on Myocardial Mitochondrial Metabolism}

Recently, studies focusing on mitochondrial integrity and function under LV unloading have been increasing. The improvement of mitochondrial dysfunction is firstly reported in patients with HF supported with long-term left ventricular assist device (LVAD) therapy (140). To elucidate the mechanisms of the clinical benefit brought by LVAD, tissue specimens were harvested from the explanted hearts at the time of transplantation or LVAD implantation. Mechanical unloading supported by LVAD increased the respiratory rate and efficiency of mitochondrial respiration on TCA cycle-derived substrates in isolated mitochondria, suggesting that mitochondrial 
metabolism is involved in the cardioprotective effect of LVAD (140). In failing human myocardium, decreased oxidation and increased accumulation of long-chain FAs in the hypertrophic heart are accompanied by increased acyl-derived intermediates, including lipotoxic ceramides that curtail cardiac function. Mechanical unloading via LVAD implantation increased the content of acyl CoA, facilitating the lipid trafficking and amelioration of the lipotoxicity (141-143). Besides, by decreasing LV afterload, LVAD reduces acyl chains required for energy production of the myocardium, which, therefore, restores the upstream acyl CoA pool (143). In the IR injury model, compared with pure reperfusion, LV unloading for $30 \mathrm{~min}$ prior to reperfusion upregulates genes associated with mitochondrial function and respiration, and maintains the integrity of mitochondria in the infarct zone (135). And tissue samples from the infarct zone with or without LV unloading following perfusion were analyzed using untargeted metabolomics. Compared with the sham control group, IR significantly reduces the levels of key metabolites related to glycolysis, glucose oxidation, fatty acid transport and oxidation, and amino acid use, while LV unloading preserves the levels of these metabolites (139). Pathway analysis also showed that, compared with reperfusion alone, LV unloading remarkably altered various pathways involved in glycolysis, the TCA cycle, and ETC function, suggesting that LV unloading preserves substrate levels for OXPHOS (139). Further functional studies showed that, compared with IR, LV unloading retains intact mitochondrial structure, including cardiolipin content and activity of ETC, including mitochondrial Complex I, and reduces oxidative stress in mitochondria isolated from the infarct zone. In conclusion, by reestablishing the oxygen supply-consumption balance, the primary clinical prognosis determinant of patients with IHD, LV mechanical unloading preserves myocardial energetics and mitochondrial function, and, therefore, limits the infarct size and improves cardiac function after AMI (Figure 2). Nevertheless, more preclinical and human studies concerning LV mechanical unloading require to be done since the panorama of substrate utilization and mitochondrial metabolism is incomplete.

\section{Metabolic Biomarkers of Myocardial Recovery Under LV Unloading}

Recently, the effects of mechanical unloading on mitochondrial metabolism in patients with progressive $\mathrm{HF}$, including ischemic and non-ischemic cardiomyopathy, have increasingly investigated to identify whether progression and improvement of $\mathrm{HF}$ are correlated with altered myocardial energetics and the metabolic process induced by mechanical unloading. In the ischemic myocardium, altered distribution of $\mathrm{CK}$ isoenzyme may disrupt energy transfer between mitochondria and the contractile apparatus. Cardiolipin is the characteristic phospholipid of the mitochondrial inner membrane whose functional status is a determinant for efficient mitochondrial coupling. Previous studies have shown that LVAD support can improve mitochondrial coupling in ischemic cardiomyopathy (ICM) hearts, and the improvement is associated with the normalization of cardiolipin composition $(140,144)$.

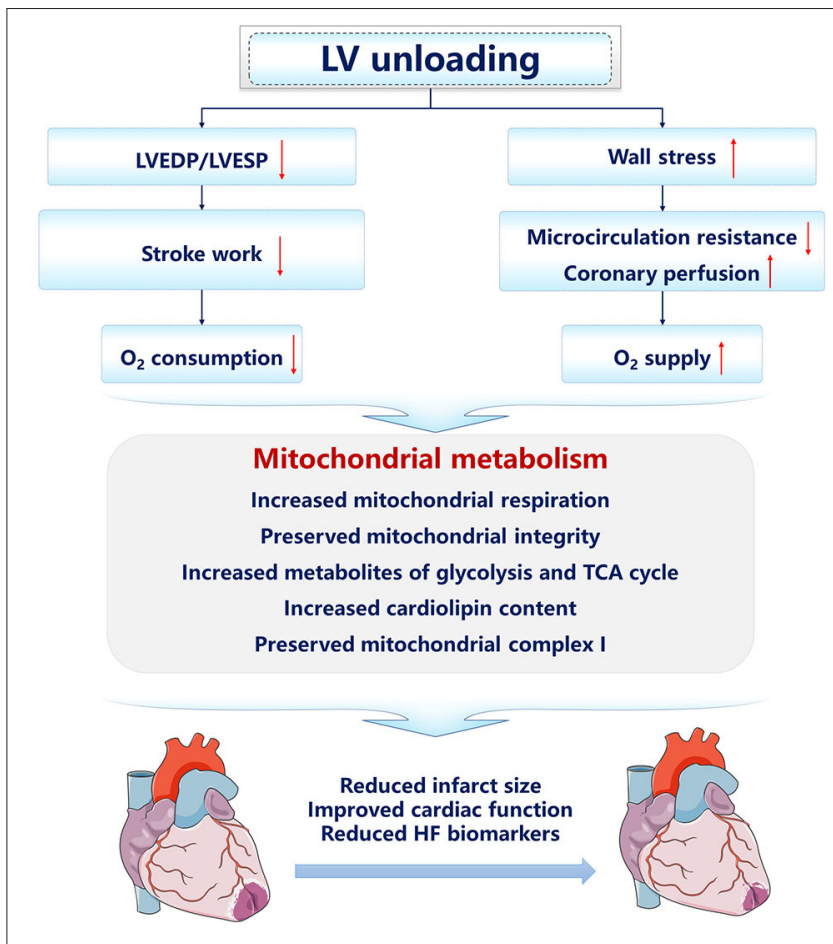

FIGURE 2 | The cardioprotective mechanisms of left ventricular unloading. The arrow facing up represents an increase, and down indicates a decrease.

In normal hearts, lactate can be converted to pyruvate by lactate dehydrogenase (LDH) to enter the TCA cycle and generate ATP, and concurrent lactate production balances the lactate consumption (145). During HF, this balance is disturbed, with an increased glycolytic pyruvate-derived lactate and a simultaneous decrease in lactate utilization (146). Diakos et al. demonstrated that glycolytic metabolites are increased in patients with post-LVAD HF without a corresponding increase of early TCA cycle intermediates, which may be attributed to poor recovery of mitochondrial oxidative capacity and volume density post-LVAD (146). Besides, the subsequent intermediates after succinyl CoA anaplerotic access and amino acids levels are increased post LVAD unloading, representing a complementary energy source that could fuel the TCA cycle and building blocks for protein synthesis (146). Especially, they revealed that patients with better myocardial recovery post LVAD seem to have improved mitochondrial function and structure with LVAD unloading. This is verified in subsequent study showing that MPC1 was lower in myocardial tissue samples of patients with HF than those of non-failing donors (147). The MPC transports pyruvate into the mitochondria for the TCA cycle and is an important modulating point, which determines whether pyruvate is oxidized or converted to lactate (148). In these patients with chronic HF, increased MPC1 abundance postLVAD is observed in responders (patients with significantly improved myocardial function after LVAD unloading) but not non-responders, suggesting defective mitochondrial pyruvate metabolism may be a primary factor contributing to their HF. 
Further animal models and in vitro cardiomyocyte models proved that inhibited mitochondrial pyruvate oxidation is closely correlated with cardiomyocyte hypertrophy.

Except for glucose oxidation, the metabolites of glycolysis may be channeled to accessory pathways of glycolysis. Clinically, LVAD implantation enhances levels of glucose and glucose6-phosphate in patients with ICM (149). Compared with post-LVAD non-responders, rate-limiting enzymes of PPP and one-carbon metabolism, which protect myocardium through producing NADPH to promote biosynthesis and antagonizing oxidative stress, are significantly increased in post-LVAD responders (150). The responders also have improved mitochondrial density and increased expression of $\alpha$-dystroglycan that maintains the extracellular matrix and cytoskeletal integrity (150).Therefore, the deleterious mitochondrial or related metabolism pathway may be underlying etiology for the cardiac response to LVAD, and specific metabolites may be utilized as cardiac prognostic biomarkers of LVAD implantation. However, concerning LVAD study on HF that includes patients with ischemic and non-ischemic cardiomyopathy, caution should be made when interpreting these results as studies have shown that metabolic processes can be used to distinguish non-ischemic dilated cardiomyopathy from ICM (151).

\section{Hemodynamics of VA-ECMO}

Cardiac arrest or cardiogenic shock (CS) occurs in $\sim 3-$ $10 \%$ of patients with AMI and is associated with about 30$50 \%$ in-hospital mortality $(152,153)$. Therefore, percutaneous circulatory support, particularly venoarterial extracorporeal membrane oxygenation (VA-ECMO), which belongs to RATo-Aterial circulatory support, is increasingly used to provide hemodynamic support for the management of AMI. Since VAECMO transfers blood from venous reservoir to the arterial system, the afterload is increased due to the retrograde flow. Thus, the volume status and native ventricular function of the patients will affect LV volume. In patients with low volume in the venous system, VA-ECMO initiation reduces the total cardiac preload and therefore LV volume (154). However, as VA-ECMO is usually applied to CS patients with venous congestion, the pressurized arterial system will increase systemic blood pressure. When the original LV function is retained, the increase in LV systolic pressure will overcome the LV afterload to enable blood ejection via aortic valve, and the increased flow and LV systolic blood pressure will not come at the cost of LV diastolic blood pressure when the LV contractility is preserved (154). In patients with LV dysfunction compromised by extensive anterior wall MI, the diminished stroke volume results in increased LV systolic and diastolic pressures because the dysfunctional LV cannot pump blood effectively to resist increased afterload and the pressurized arterial tree (110). The elevated LV afterload increases wall stress of LV and left atrial and therefore increases oxygen demand, which finally hinders myocardial recovery (155). The extra LV afterload and insufficient LV mechanical unloading under VA-ECMO may lead to severe complications, including LV stasis, thrombosis, pulmonary edema, and ventricular dilatationinduced ischemia, all of which increase mortality $(156,157)$.
Particularly, ECMO can reduce both LV end diastolic volume and LV systolic wall stress of the normal hearts but increase LV wall stress in ischemic hearts (158).

To decompress the LV and attenuate increased afterload via elevating forward flow, the VA-ECMO is combined with other mechanical devices in clinical practices (159). In a retrospective cohort from America, almost $60 \%$ of AMI admissions had a second temporary MCS device besides ECMO, and 30.3\% of them were placed concomitantly (160). Previous meta-analysis suggests that ECMO + IABP strategy achieves lower mortality compared with ECMO alone in CS of AMI (161). Compared with that of patients with VA-ECMO alone, the mortality was lower in patients with combined Impella and VA-ECMO (162, 163). Besides, LV unloading done early (within $12 \mathrm{~h}$ ) seems to be correlated with an increased rate of successful weaning and decreased short-term mortality (164). Recent studies have suggested that combined utilization of Impella to decompress the LV with ECMO reduced the mortality rate of CS (154). However, as VA-ECMO support systemic perfusion at the risk of increasing LV load, available data on VA-ECMO utilization in AMI are limited, and most of them are retrospective. There is an urgent need to conduct randomized controlled trials (RCT) to investigate the application of VA-ECMO in AMI and its effects on infarct size as well as long-term prognosis.

\section{VA-ECMO on Mitochondrial Metabolism of Normal Hearts}

As recovery of myocardial function is the primary prerequisite for weaning from the ECMO circuit, it is necessary to fully clarify the effects of changing energy equilibrium and substrate utilization of the heart under ECMO support. Of all these factors, hemodynamics of the heart during ECMO cause changes in substrate metabolism. Even if no direct myocardial damage has occurred before, ECMO can cause cardiac arrest. Therefore, the previously uninjured and unenlarged heart is used to define the effect of ECMO on substrate use and myocardial energetics to exclude the interferences of hypoxia, ischemia, or reperfusion. In infants, systemic studies have shown that ECMO promotes the leucine oxidation and, at the same time, increases the protein degradation rate, leading to negative protein balance and related skeletal muscle atrophy (165). The BCAA undergoes catabolism instead of being incorporated into protein synthesis, providing a carbon substrate for oxidation in the TCA cycle (166). However, evidence concerning heartspecific adaptions in the metabolic process is rare. In an immature swine model, ECMO shifts myocardial metabolic processes toward positive protein balance by preserving BCAA for protein synthesis instead of promoting their oxidation (167). Because ECMO significantly reduces myocardial oxygen consumption, the overall LV leucine oxidation rate is reduced compared with the increase observed in the whole-bodyinfant study. Besides, pyruvate utilization seems to prevent insulin resistance, further preserving BCAA from oxidation. Simultaneously, ECMO promotes the oxidation of long chain FAs oxidation and inhibits lactate oxidation (168). This metabolic shift during ECMO appears to be explained by rapid 
upregulation of PDH kinase-4 (PDK4) protein. As demonstrated by Randle et al. (169), $\mathrm{PDH}$ is phosphorylated and inhibited by PDK4, thereby reciprocally increasing FAO via acyl-CoA dehydrogenases. ECMO also increased the relative flux from lactate to alanine, further supporting the inhibitory effect of PDK4 on PDH (168). In a VA-ECMO immature piglet model, weaning from ECMO circuits induces a sudden increase in cardiac work and myocardial oxygen consumption, which is associated with shifts in substrate utilization and pathways contribution. The exogenous pyruvate is favored vs. those derived from glycolysis, and acetyl-CoA is generated from endogenous substrate, presumably fatty acids and ketones rather than pyruvate decarboxylation (170). In conclusion, in normal hearts, ECMO preserved positive protein balance via promoting the FAO and pyruvate oxidation, and successful weaning will not increase the oxidation of amino acids, nor will it adversely affect the rate of protein synthesis.

\section{VA-ECMO on Mitochondrial Metabolism of Ischemic Hearts}

To determine whether VA-ECMO protects the ischemic heart through ameliorating metabolic abnormalities induced by IR, metabolites of neonatal piglets that undergo coronary occlusion for $30 \mathrm{~min}$, followed by ECMO support, have been analyzed (170). The ratio of phosphocreatine to adenosine triphosphate (PCr/ATP) is analyzed as a surrogate for mitochondrial oxidative capacity (171), and it falls when ATP demand nears or exceeds the maximal ATP production rate. Files demonstrated that, compared with the preischemic level, IR significantly decreased the PCr/ATP by $>50 \%$, and ECMO after injury returns the PCr/ATP to near preischemic levels, yet this effect is lost during weaning (172). This suggests that mitochondrial ATP synthesis operates near maximal capacity, as weaning ECMO elevates the energy requirement and disrupts the PCr/ATP. Along with the impaired mitochondrial respiration, IR significantly expanded the intracellular pyruvate pool, and shifted pyruvate from oxidation toward anaerobic glycolysis, indicting by reducing pyruvate decarboxylation (PDC) relative to citric acid cycle (CAC) flux (172). ECMO significantly increased the pyruvate fractional contribution, while weaning followed a similar pattern (172). Besides, the CAC flux is also impaired after ischemia and during reloading. IR did not alter the level of citrate and $\alpha-K G$ but did substantially expand the pools of distal CAC intermediates succinate, fumarate, and malate. The accumulation of succinate is induced by forward flux from $\alpha-K G$ with partial inhibition of SDH within mitochondrial complex II. The high concentrations of succinate might readjust activity of dehydrogenase and produce fumarate and malate at a higher concentration. Alternatively, metabolic analysis suggests that the expansion of oxaloacetate and malate was partially through the anaplerotic entry of pyruvate. As demonstrated previously, IR can promote the carboxylation of anaplerotic pyruvate, which is converted to oxaloacetate by its carboxylase or converted to malate by malic enzyme $(173,174)$. The metabolic impairments, including the reduced $\mathrm{PDC}$ rate and accumulated succinate, also recurred with ECMO weaning, implying that limited OXPHOS due to insufficient acetyl-CoA and disrupted cycling of CAC impaired weaning.

In another adult swine model subjected to LAD occlusion, VAECMO was activated followed with 180-min LAD reperfusion, and the hemodynamic parameters were detected. Compared with the group of IR injury, ECMO initiation reduces right atrial RA pressure and LV stroke work but increases the infarct area normalized to the risk area and the infarct area normalized to the total LV area (139). Metabolic data based on unbiased and blinded analysis show that LV unloading by ECMO before reperfusion does not improve the use of myocardial energy substrates, nor can it preserve mitochondrial structure, including cardiolipin content induced by IR injury. Further functional analysis of the mitochondria of infarct zone showed that reperfusion that followed ECMO support also has impaired ETC, including mitochondrial Complexes I, II, and III, and increased oxidative stress with IR. Therefore, despite the fact that ECMO does reduce LV stroke work, it increases the infarct area and fails to protect myocardial mitochondrial and energy metabolism. It should be noted that, except for the hemodynamic effects, the large surface areas of extracorporeal circuits can activate neutrophils, which has already been demonstrated in patients with CS, and the increased circulating neutrophils can mediate reperfusion injury (175). In addition, the data that bypass the left atrium to the femoral artery without an oxygenator can reduce LV stroke work and infarct size also proves this point (133).

In addition to immune alterations, the discrepancies of the two preclinical studies may be attributed to differences between experimental models, such as animal maturity, duration and severity of ischemia, timing of ECMO initiation, flow of ECMO, timing of sampling, etc. Besides, the native cardiac volume and hemodynamic parameters are to be continuously monitored, since they are also correlated with tissue perfusion. As successful weaning of ECMO is based on end-organ function and myocardial recovery, which includes myocardial energetics and substrate utilization, metabolic pathways may be promising therapeutic targets in future clinical practices. Moreover, combined with profound hemodynamic monitoring, metabolites may be used as biomarkers to develop more comprehensive and superior algorithms or models for evaluating myocardial functional recovery.

\section{CONCLUSION}

This study summarizes substrate use and metabolic alterations during the process of IHD, with an emphasis on IR injury and HF. Although numerous metabolic processes outlined here are ideal drug targets for IR injury, further pieces of research are still needed to gain full insights into the underlying mechanism. Particularly, by unloading the ischemic heart and establishing the oxygen supply-demand balance, MCS is 
promising in reducing IR injury and improving cardiac function. However, their effects on mitochondrial metabolism and cardiac improvement, especially VA-ECMO, still vary under different conditions. Therefore, more studies are needed to elucidate whether MCS has a beneficial impact on cardiac function and long-term prognosis.

\section{AUTHOR CONTRIBUTIONS}

MJ wrote the draft of the manuscript and prepared the figures. XX reviewed the manuscript. FC and YW proposed the original idea and reviewed the manuscript. All the

\section{REFERENCES}

1. Nowbar AN, Gitto M, Howard JP, Francis DP, Al-Lamee R. Mortality from ischemic heart disease. Circulation. (2019) 12:5375. doi: 10.1161/CIRCOUTCOMES.118.005375

2. Kaski J, Crea F, Gersh BJ, Camici PG. Reappraisal of ischemic heart disease. Circulation. (2018) 138:146380. doi: 10.1161/CIRCULATIONAHA.118.031373

3. Lee L. Metabolic manipulation in ischaemic heart disease, a novel approach to treatment. Eur Heart J. (2004) 25:634-41. doi: 10.1016/j.ehj.2004.02.018

4. Lopaschuk GD, Karwi QG, Tian R, Wende AR, Abel ED. Cardiac energy metabolism in heart failure. Circ Res. (2021) 128:1487-513. doi: 10.1161/CIRCRESAHA.121.318241

5. Faadielessop M, Opie L. Metabolic therapy for heart failure. Eur Heart J. (2004) 25:1765-8. doi: 10.1016/j.ehj.2004.08.019

6. Lopaschuk GD, Ussher JR, Folmes CDL, Jaswal JS, Stanley WC. Myocardial fatty acid metabolism in health and disease. Physiol Rev. (2010) 90:20758. doi: 10.1152/physrev.00015.2009

7. Gorge G, Chatelain P, Schaper J, Lerch R. Effect of increasing degrees of ischemic injury on myocardial oxidative metabolism early after reperfusion in isolated rat hearts. Circ Res. (1991) 68:1681-92. doi: 10.1161/01.RES.68.6.1681

8. Zuurbier CJ, Bertrand L, Beauloye CR, Andreadou I, Ruiz-Meana M, Jespersen NR, et al. Cardiac metabolism as a driver and therapeutic target of myocardial infarction. J Cell Mol Med. (2020) 24:593754. doi: $10.1111 / \mathrm{jcmm} .15180$

9. Zuurbier CJ, Ince C. Post-ischaemic changes in the response time of oxygen consumption to demand in the isolated rat heart are mediated partly by calcium and glycolysis. Pflugers Arch. (2002) 443:90816. doi: $10.1007 / \mathrm{s} 00424-001-0744-2$

10. Benzi RH, Lerch R. Dissociation between contractile function and oxidative metabolism in postischemic myocardium. Attenuation by ruthenium red administered during reperfusion. Circ Res. (1992) 71:56776. doi: 10.1161/01.RES.71.3.567

11. Stanley WC, Lopaschuk GD, Hall JL, Mccormack JG. Regulation of myocardial carbohydrate metabolism under normal and ischaemic conditions. Potential for pharmacological interventions. Cardiovasc Res. (1997) 33:243-57. doi: 10.1016/S0008-6363(96)00245-3

12. Tanaka-Esposito C, Chen Q, Lesnefsky EJ. Blockade of electron transport before ischemia protects mitochondria and decreases myocardial injury during reperfusion in aged rat hearts. Transl Res. (2012) 160:20716. doi: $10.1016 /$ j.trsl.2012.01.024

13. Turer AT, Stevens RD, Bain JR, Muehlbauer MJ, van der Westhuizen J, Mathew JP, et al. Metabolomic profiling reveals distinct patterns of myocardial substrate use in humans with coronary artery disease or left ventricular dysfunction during surgical Ischemia/Reperfusion. Circulation. (2009) 119:1736-46. doi: 10.1161/CIRCULATIONAHA.108.81 6116 authors read and approved the final submitted version of the manuscript.

\section{FUNDING}

This work was supported by Beijing Municipal Natural Science Foundation (7202189), Project of National Clinical Research Center for Geriatric Disease (NCRCG-PLAGH-2019024), The National Key Research Program of China (2018YFC0116305), The National Natural Science Foundation of China (grant nos. 91939303 and 81820108019), and Major Program of Central Health Committee (2020ZD05).

14. Montessuit C, Lerch R. Regulation and dysregulation of glucose transport in cardiomyocytes. Biochimica Biophysica Acta. (2013) 1833:848-56. doi: 10.1016/j.bbamcr.2012.08.009

15. Shi T, Papay RS, Perez DM. Alpha1A-Adrenergic receptor prevents cardiac ischemic damage through PKCdelta/GLUT1/4mediated glucose uptake. J Recept Signal Transduct Res. (2016) 36:261-70. doi: 10.3109/10799893.2015.1091475

16. Li X, Liu Y, Ma H, Guan Y, Cao Y, Tian Y, et al. Enhancement of glucose metabolism via PGC-1 $\alpha$ participates in the cardioprotection of chronic intermittent hypobaric hypoxia. Front Physiol. (2016) 7:219. doi: 10.3389/fphys.2016.00219

17. Liu X, Zeng J, Yang Y, Qi C, Xiong T, Wu G, et al. DRD4 mitigates myocardial Ischemia/Reperfusion injury in association with PI3K/AKT mediated glucose metabolism. Front Pharmacol. (2021) 11:619426. doi: 10.3389/fphar.2020.619426

18. Tian R, Abel ED. Responses of GLUT4-deficient hearts to ischemia underscore the importance of glycolysis. Circulation. (2001) 103:29616. doi: 10.1161/01.CIR.103.24.2961

19. Omar MA, Verma S, Clanachan AS. Adenosine-mediated inhibition of $5^{\prime}$-AMP-activated protein kinase and p38 mitogen-activated protein kinase during reperfusion enhances recovery of left ventricular mechanical function. J Mol Cell Cardiol. (2012) 52:1308-18. doi: 10.1016/j.yjmcc.2012.03.014

20. Heidrich F, Schotola H, Popov AF, Sohns C, Schuenemann J, Friedrich M, et al. AMPK - activated protein kinase and its role in energy metabolism of the heart. Curr Cardiol Rev. (2010) 6:337-42. doi: 10.2174/157340310793566073

21. Fernández-Caggiano M, Prysyazhna O, Barallobre-Barreiro J, Calviñosantos R, Aldama López G, Generosa Crespo-Leiro M, et al. Analysis of mitochondrial proteins in the surviving myocardium after ischemia identifies mitochondrial pyruvate carrier expression as possible mediator of tissue viability. Mol Cell Proteomics. (2016) 15:246-55. doi: 10.1074/mcp.M115.051862

22. Kudo N, Barr AJ, Barr RL, Desai S, Lopaschuk GD. High rates of fatty acid oxidation during reperfusion of ischemic hearts are associated with a decrease in malonyl-CoA levels due to an increase in 5'-AMP-activated protein kinase inhibition of acetyl-CoA carboxylase. J Biol Chem. (1995) 270:17513-20. doi: 10.1074/jbc.270.29.17513

23. Chouchani ET, Methner C, Nadtochiy SM, Logan A, Pell VR, Ding S, et al. Cardioprotection by S-nitrosation of a cysteine switch on mitochondrial complex I. Nat Med. (2013) 19:753-9. doi: 10.1038/nm.3212

24. Garland PB, Randle PJ, Newsholme EA. Citrate as an intermediary in the inhibition of phosphofructokinase in rat heart muscle by fatty acids, ketone bodies, pyruvate, diabetes, and starvation. Nature. (1963) 200:16970. doi: 10.1038/200169a0

25. Heather LC, Clarke K. Metabolism, hypoxia and the diabetic heart. J Mol Cell Cardiol. (2011) 50:598-605. doi: 10.1016/j.yjmcc.2011.0 1.007 
26. Lopaschuk GD, Spafford MA, Davies NJ, Wall SR. Glucose and palmitate oxidation in isolated working rat hearts reperfused after a period of transient global ischemia. Circ Res. (1990) 66:546-53. doi: 10.1161/01.RES.66.2.546

27. Terrand J, Papageorgiou I, Rosenblatt-Velin N, Lerch R. Calcium-mediated activation of pyruvate dehydrogenase in severely injured postischemic myocardium. Am J Physiol Heart Circ Physiol. (2001) 281:H72230. doi: 10.1152/ajpheart.2001.281.2.H722

28. Lerch R, Tamm C, Papageorgiou I, Benzi RH. Myocardial fatty acid oxidation during ischemia and reperfusion. Mol Cell Biochem. (1992) 116:1039. doi: 10.1007/BF01270576

29. Lewandowski ED, White LT. Pyruvate dehydrogenase influences postischemic heart function. Circulation. (1995) 91:20719. doi: 10.1161/01.CIR.91.7.2071

30. Wang P, Lloyd SG, Chatham JC. Impact of high glucose/high insulin and dichloroacetate treatment on carbohydrate oxidation and functional recovery after low-flow ischemia and reperfusion in the isolated perfused rat heart. Circulation. (2005) 111:206672. doi: 10.1161/01.CIR.0000162466.06150.D4

31. Luptak I, Yan J, Cui L, Jain M, Liao R, Tian R. Long-term effects of increased glucose entry on mouse hearts during normal aging and ischemic stress. Circulation. (2007) 116:9019. doi: 10.1161/CIRCULATIONAHA.107.691253

32. Mansor LS, Sousa FM, Yea G, Coumans WA, West JA, Kerr M, et al. Inhibition of sarcolemmal FAT/CD36 by sulfo-N-succinimidyl oleate rapidly corrects metabolism and restores function in the diabetic heart following hypoxia/reoxygenation. Cardiovasc Res. (2017) 113:73748. doi: $10.1093 / \mathrm{cvr} / \mathrm{cvx} 045$

33. Mccormack JG, Barr RL, Wolff AA, Lopaschuk GD. Ranolazine stimulates glucose oxidation in normoxic, ischemic, and reperfused ischemic rat hearts. Circulation. (1996) 93:135-42. doi: 10.1161/01.CIR.93.1.135

34. Ussher JR, Wang W, Gandhi M, Keung W, Samokhvalov V, Oka $\mathrm{T}$, et al. Stimulation of glucose oxidation protects against acute myocardial infarction and reperfusion injury. Cardiovasc Res. (2012) 94:359-69. doi: 10.1093/cvr/cvs129

35. Pham V, Zhang W, Chen V, Whitney T, Yao J, Froese D, et al. Design and synthesis of novel pyridoxine 5'-phosphonates as potential antiischemic agents. J Med Chem. (2003) 46:3680-7. doi: 10.1021/jm0300678

36. Heywood SE, Richart AL, Henstridge DC, Alt K, Kiriazis H, Zammit $\mathrm{C}$, et al. High-density lipoprotein delivered after myocardial infarction increases cardiac glucose uptake and function in mice. Sci Transl Med. (2017) 9:aam6084. doi: 10.1126/scitranslmed.aam6084

37. Doenst T, Nguyen TD, Abel ED. Cardiac metabolism in heart failure. Circ Res. (2013) 113:709-24. doi: 10.1161/CIRCRESAHA.113.300376

38. Ritterhoff J, Tian R. Metabolism in cardiomyopathy: every substrate matters. Cardiovasc Res. (2017) 113:411-21. doi: 10.1093/cvr/cvx017

39. Griffiths HR, Gao D, Pararasa C. Redox regulation in metabolic programming and inflammation. Redox Biol. (2017) 12:507. doi: 10.1016/j.redox.2017.01.023

40. Ou W, Liang Y, Qing Y, Wu W, Xie M, Zhang Y, et al. Hypoxic acclimation improves cardiac redox homeostasis and protects heart against ischemiareperfusion injury through upregulation of O-GlcNAcylation. Redox Biol. (2021) 43:101994. doi: 10.1016/j.redox.2021.101994

41. Ellis JM, Frahm JL Li LO, Coleman RA. Acyl-coenzyme a synthetases in metabolic control. CurrOpinLipidol. (2010) 21:212-7. doi: 10.1097/MOL.0b013e32833884bb

42. Hopkins TA, Dyck JRB, Lopaschuk GD. AMP-activated protein kinase regulation of fatty acid oxidation in the ischaemic heart. Biochem Soc T. (2003) 31:207. doi: 10.1042/bst0310207

43. Shu H, Peng Y, Hang W, Nie J, Zhou N, Wang DW. The role of CD36 in cardiovascular disease. Cardiovasc Res. (2020) 2020:cvaa319. doi: 10.1093/cvr/cvaa319

44. Ussher JR, Lopaschuk GD. The malonyl CoA axis as a potential target for treating ischaemic heart disease. Cardiovasc Res. (2008) 79:25968. doi: $10.1093 / \mathrm{cvr} / \mathrm{cvn} 130$

45. Folmes CDL, Sowah D, Clanachan AS, Lopaschuk GD. High rates of residual fatty acid oxidation during mild ischemia decrease cardiac work and efficiency. J Mol Cell Cardiol. (2009) 47:142-8. doi: 10.1016/j.yjmcc.2009.03.005
46. Heather LC, Pates KM, Atherton HJ, Cole MA, Ball DR, Evans RD, et al. Differential translocation of the fatty acid transporter, FAT/CD36, and the glucose transporter, GLUT4, coordinates changes in cardiac substrate metabolism during ischemia and reperfusion. Circulation. (2013) 6:105866. doi: 10.1161/CIRCHEARTFAILURE.112.000342

47. Tominaga H, Katoh H, Odagiri K, Takeuchi Y, Kawashima H, Saotome $\mathrm{M}$, et al. Different effects of palmitoyl-1-carnitine and palmitoyl-CoA on mitochondrial function in rat ventricular myocytes. Am J Physiol-Heart C. (2008) 295:H105-12. doi: 10.1152/ajpheart.01307.2007

48. Liepinsh E, Makrecka-Kuka M, Volska K, Kuka J, Makarova E, Antone $\mathrm{U}$, et al. Long-chain acylcarnitines determine ischaemia/reperfusioninduced damage in heart mitochondria. Biochem J. (2016) 473:1191202. doi: 10.1042/BCJ20160164

49. Tomanek RJ. Myocardial ischemia and infarction. In: Tomanek RJ, editor, Coronary Vasculature: Development, Structure-Function, and Adaptations. Boston, MA: Springer US (2013). p. 189220. doi: 10.1007/978-1-4614-4887-7_10

50. Wang W, Zhang L, Battiprolu PK, Fukushima A, Nguyen K, Milner $\mathrm{K}$, et al. Malonyl CoA decarboxylase inhibition improves cardiac function Post-Myocardial infarction. JACC. (2019) 4:385-400. doi: 10.1016/j.jacbts.2019.02.003

51. Dyck JRB, Cheng J, Stanley WC, Barr R, Chandler MP, Brown $S$, et al. Malonyl coenzyme a decarboxylase inhibition protects the ischemic heart by inhibiting fatty acid oxidation and stimulating glucose oxidation. Circ Res. (2004) 94:19569. doi: 10.1161/01.RES.0000129255.195 $69.8 \mathrm{f}$

52. Liepinsh E, Makrecka-Kuka M, Kuka J, Vilskersts R, Makarova E, Cirule $\mathrm{H}$, et al. Inhibition of L-carnitine biosynthesis and transport by methyl $-\gamma$ butyrobetaine decreases fatty acid oxidation and protects against myocardial infarction. Brit J Pharmacol. (2015) 172:1319-32. doi: 10.1111/bph.1 3004

53. Liu Z, Chen J, Huang H, Kuznicki M, Zheng S, Sun W, et al. The protective effect of trimetazidine on myocardial ischemia/reperfusion injury through activating AMPK and ERK signaling pathway. Metabolism. (2016) 65:12230. doi: 10.1016/j.metabol.2015.10.022

54. Hu H, Li X, Ren D, Tan Y, Chen J, Yang L, et al. The cardioprotective effects of carvedilol on ischemia and reperfusion injury by AMPK signaling pathway. Biomed Pharmacother. (2019) 117:109106. doi: 10.1016/j.biopha.2019.109106

55. Liu Q, Docherty JC, Rendell JCT, Clanachan AS, Lopaschuk GD. High levels of fatty acids delay the recovery of intracellular $\mathrm{pH}$ and cardiac efficiency in post-ischemic hearts by inhibiting glucose oxidation. J Am Coll Cardiol. (2002) 39:718-25. doi: 10.1016/S0735-1097(01)01803-4

56. Cotter DG, Schugar RC, Crawford PA. Ketone body metabolism and cardiovascular disease. Am J Physiol-Heart C. (2013) 304:H1060-76. doi: 10.1152/ajpheart.00646.2012

57. Ho KL, Zhang L, Wagg C, Al Batran R, Gopal K, Levasseur J, et al. Increased ketone body oxidation provides additional energy for the failing heart without improving cardiac efficiency. Cardiovasc Res. (2019) 115:160616. doi: $10.1093 / \mathrm{cvr} / \mathrm{cvz} 045$

58. Anorek M, Hodyc D, Aedivý V, Durišová J, Skoumalová A, Wilhelm J, et al. Short-term fasting reduces the extent of myocardial infarction and incidence of reperfusion arrhythmias in rats. Physiol Res. (2012) 2012:56774. doi: 10.33549/physiolres. 932338

59. Al-Zaid NS, Dashti HM, Mathew TC, Juggi JS. Low carbohydrate ketogenic diet enhances cardiac tolerance to global ischaemia. Acta Cardiol. (2007) 62:381-9. doi: 10.2143/AC.62.4.2022282

60. Wang P, Tate JM, Lloyd SG. Low carbohydrate diet decreases myocardial insulin signaling and increases susceptibility to myocardial ischemia. Life Sci. (2008) 83:836-44. doi: 10.1016/j.lfs.2008.09.024

61. Zou Z, Sasaguri S, Rajesh KG, Suzuki R. Dl-3-Hydroxybutyrate administration prevents myocardial damage after coronary occlusion in rat hearts. Am J Physiol-Heart C. (2002) 283:H196874. doi: 10.1152/ajpheart.00250.2002

62. Shimazu T, Hirschey MD, Newman J, He W, Shirakawa K, Le Moan $\mathrm{N}$, et al. Suppression of oxidative stress by -Hydroxybutyrate, an endogenous histone deacetylase inhibitor. Science. (2013) 339:211-4. doi: 10.1126/science.1227166 
63. Puchalska P, Crawford PA. Multi-dimensional roles of ketone bodies in fuel metabolism, signaling, and therapeutics. Cell Metab. (2017) 25:26284. doi: 10.1016/j.cmet.2016.12.022

64. Yu Y, Yu Y, Zhang Y, Zhang Z, An W, Zhao X. Treatment with D- $\beta$ hydroxybutyrate protects heart from ischemia/reperfusion injury in mice. Eur J Pharmacol. (2018) 829:121-8. doi: 10.1016/j.ejphar.2018.04.019

65. Arima Y, Izumiya $Y$, Ishida T, Takashio S, Ishii M, Sueta D, et al. Myocardial ischemia suppresses ketone body utilization. J Am Coll Cardiol. (2019) 73:246-7. doi: 10.1016/j.jacc.2018.10.040

66. Aubert G, Martin OJ, Horton JL, Lai L, Vega RB, Leone TC, et al. The failing heart relies on ketone bodies as a fuel. Circulation. (2016) 133:698-705. doi: 10.1161/CIRCULATIONAHA.115.0 17355

67. Uchihashi M, Hoshino A, Okawa Y, Ariyoshi M, Kaimoto S, Tateishi S, et al. Cardiac-Specific bdh1 overexpression ameliorates oxidative stress and cardiac remodeling in pressure overload-induced heart failure. Circulation. (2017) 10:4417. doi: 10.1161/CIRCHEARTFAILURE.117.004417

68. Horton JL, Davidson MT, Kurishima C, Vega RB, Powers JC, Matsuura TR, et al. The failing heart utilizes 3-hydroxybutyrate as a metabolic stress defense. JCI Insight. (2019) 4:124079. doi: 10.1172/jci.insight.124079

69. Yurista SR, Silljé HHW, Oberdorf Maass SU, Schouten EM, Pavez Giani MG, Hillebrands JL, et al. Sodium-glucose co-transporter 2 inhibition with empagliflozin improves cardiac function in non-diabetic rats with left ventricular dysfunction after myocardial infarction. Eur J Heart Fail. (2019) 21:862-73. doi: 10.1002/ejhf.1473

70. Selvaraj S, Kelly DP, Margulies KB. Implications of altered ketone metabolism and therapeutic ketosis in heart failure. Circulation. (2020) 141:180012. doi: 10.1161/CIRCULATIONAHA.119.045033

71. Huang Y, Zhou M, Sun H, Wang Y. Branched-chain amino acid metabolism in heart disease: an epiphenomenon or a real culprit? Cardiovasc Res. (2011) 90:220-3. doi: $10.1093 / \mathrm{cvr} / \mathrm{cvr} 070$

72. Bhattacharya S, Granger CB, Craig D, Haynes C, Bain J, Stevens RD, et al. Validation of the association between a branched chain amino acid metabolite profile and extremes of coronary artery disease in patients referred for cardiac catheterization. Atherosclerosis. (2014) 232:1916. doi: 10.1016/j.atherosclerosis.2013.10.036

73. Santos-Gallego CG, Requena-Ibanez JA, San Antonio R, Ishikawa K, Watanabe S, Picatoste B, et al. Empagliflozin ameliorates adverse left ventricular remodeling in nondiabetic heart failure by enhancing myocardial energetics. J Am Coll Cardiol. (2019) 73:1931-44. doi: 10.1016/j.jacc.2019.01.056

74. Wang W, Zhang F, Xia Y, Zhao S, Yan W, Wang H, et al. Defective branched chain amino acid catabolism contributes to cardiac dysfunction and remodeling following myocardial infarction. Am J Physiol-Heart C. (2016) 311:H1160-9. doi: 10.1152/ajpheart.00114.2016

75. Karwi QG, Zhang L, Wagg CS, Wang W, Ghandi M, Thai D, et al. Targeting the glucagon receptor improves cardiac function and enhances insulin sensitivity following a myocardial infarction. Cardiovasc Diabetol. (2019) 18:4. doi: 10.1186/s12933-019-0806-4

76. Li T, Zhang Z, Kolwicz SC, Abell L, Roe ND, Kim M, et al. Defective Branched-Chain amino acid catabolism disrupts glucose metabolism and sensitizes the heart to Ischemia-Reperfusion injury. Cell Metab. (2017) 25:374-85. doi: 10.1016/j.cmet.2016.11.005

77. Jang C, Oh SF, Wada S, Rowe GC, Liu L, Chan MC, et al. A branched-chain amino acid metabolite drives vascular fatty acid transport and causes insulin resistance. Nat Med. (2016) 22:421-6. doi: 10.1038/nm.4057

78. Li Y, Xiong Z, Yan W, Gao E, Cheng H, Wu G, et al. Branched chain amino acids exacerbate myocardial ischemia/reperfusion vulnerability via enhancing GCN2/ATF6/PPAR- $\alpha$ pathway-dependent fatty acid oxidation. Theranostics. (2020) 10:5623-40. doi: 10.7150/thno.44836

79. Lu G, Sun H, She P, Youn J, Warburton S, Ping P, et al. Protein phosphatase $2 \mathrm{Cm}$ is a critical regulator of branched-chain amino acid catabolism in mice and cultured cells. J Clin Invest. (2009) 119:1678-87. doi: 10.1172/JCI38151

80. Lian K, Guo X, Wang Q, Liu Y, Wang RT, Gao C, et al. PP2Cm overexpression alleviates $\mathrm{MI} / \mathrm{R}$ injury mediated by a BCAA catabolism defect and oxidative stress in diabetic mice. Eur J Pharmacol. (2020) 866:172796. doi: 10.1016/j.ejphar.2019.172796
81. Dong W, Zhou M, Dong M, Pan B, Liu Y, Shao J, et al. Keto acid metabolites of branched-chain amino acids inhibit oxidative stress-induced necrosis and attenuate myocardial ischemia-reperfusion injury. J Mol Cell Cardiol. (2016) 101:90-8. doi: 10.1016/j.yjmcc.2016.11.002

82. Lesnefsky EJ, Chen Q, Moghaddas S, Hassan MO, Tandler B, Hoppel CL. Blockade of electron transport during ischemia protects cardiac mitochondria. J Biol Chem. (2004) 279:479617. doi: $10.1074 /$ jbc.M409720200

83. Sun H, Olson KC, Gao C, Prosdocimo DA, Zhou M, Wang Z, et al. Catabolic defect of Branched-Chain amino acids promotes heart failure. Circulation. (2016) 133:2038-49. doi: 10.1161/CIRCULATIONAHA.115.020226

84. Chouchani ET, Pell VR, Gaude E, Aksentijević D, Sundier SY, Robb EL, et al. Ischaemic accumulation of succinate controls reperfusion injury through mitochondrial ROS. Nature (London). (2014) 515:4315. doi: 10.1038/nature13909

85. Zhang J, Wang YT, Miller JH, Day MM, Munger JC, Brookes PS. Accumulation of succinate in cardiac ischemia primarily occurs via canonical krebs cycle activity. Cell Rep. (2018) 23:2617-28. doi: 10.1016/j.celrep.2018.04.104

86. Dambrova M, Zuurbier CJ, Borutaite V, Liepinsh E, Makrecka-Kuka M. Energy substrate metabolism and mitochondrial oxidative stress in cardiac ischemia/reperfusion injury. Free Radical Bio Med. (2021) 165:2437. doi: 10.1016/j.freeradbiomed.2021.01.036

87. Andrienko TN, Pasdois P, Pereira GC, Ovens MJ, Halestrap AP. The role of succinate and ROS in reperfusion injury - a critical appraisal. J Mol Cell Cardiol. (2017) 110:1-14. doi: 10.1016/j.yjmcc.2017.06.016

88. Chouchani ET, Pell VR, James AM, Work LM, Saeb-Parsy K, Frezza C, et al. Unifying mechanism for mitochondrial superoxide production during ischemia-reperfusion injury. Cell Metab. (2016) 23:254-63. doi: 10.1016/j.cmet.2015.12.009

89. Mills EL, Pierce KA, Jedrychowski MP, Garrity R, Winther S, Vidoni $\mathrm{S}$, et al. Accumulation of succinate controls activation of adipose tissue thermogenesis. Nature. (2018) 560:102-6. doi: 10.1038/s41586-018-0353-2

90. Kohlhauer M, Dawkins S, Costa ASH, Lee R, Young T, Pell VR, et al. Metabolomic profiling in acute ST-segment-elevation myocardial infarction identifies succinate as an early marker of human ischemia-reperfusion injury. J Am Heart Assoc. (2018) 7:7546. doi: 10.1161/JAHA.117.007546

91. Kula-Alwar D, Prag HA, Krieg T. Targeting succinate metabolism in Ischemia/Reperfusion injury. Circulation. (2019) 140:196870. doi: 10.1161/CIRCULATIONAHA.119.042791

92. Valls-Lacalle L, Barba I, Miró-Casas E, Alburquerque-Béjar JJ, RuizMeana M, Fuertes-Agudo M, et al. Succinate dehydrogenase inhibition with malonate during reperfusion reduces infarct size by preventing mitochondrial permeability transition. Cardiovasc Res. (2016) 109:37484. doi: $10.1093 / \mathrm{cvr} / \mathrm{cvv} 279$

93. Bae J, Salamon RJ, Brandt EB, Paltzer WG, Zhang Z, Britt EC, et al. Malonate promotes adult cardiomyocyte proliferation and heart regeneration. Circulation. (2021) 143:197386. doi: 10.1161/CIRCULATIONAHA.120.049952

94. Li P, Ge J, Li H. Lysine acetyltransferases and lysine deacetylases as targets for cardiovascular disease. Nat Rev Cardiol. (2020) 17:96115. doi: 10.1038/s41569-019-0235-9

95. Boylston JA, Sun J, Chen Y, Gucek M, Sack MN, Murphy E. Characterization of the cardiac succinylome and its role in ischemia-reperfusion injury. J Mol Cell Cardiol. (2015) 88:73-81. doi: 10.1016/j.yjmcc.2015.09.005

96. Porter GA, Urciuoli WR, Brookes PS, Nadtochiy SM. SIRT3 deficiency exacerbates ischemia-reperfusion injury: implication for aged hearts. Am J Physiol Heart Circulat Physiol. (2014) 306:H1602-9. doi: 10.1152/ajpheart.00027.2014

97. Xu Y, Zhang S, Rong J, Lin Y, Du L, Wang Y, et al. Sirt3 is a novel target to treat sepsis induced myocardial dysfunction by acetylated modulation of critical enzymes within cardiac tricarboxylic acid cycle. Pharmacol Res. (2020) 159:104887. doi: 10.1016/j.phrs.2020.104887

98. Davidson MT, Grimsrud PA, Lai L, Draper JA, Fisher-Wellman KH, Narowski TM, et al. Extreme acetylation of the cardiac mitochondrial proteome does not promote heart failure. Circ Res. (2020) 127:1094108. doi: 10.1161/CIRCRESAHA. 120.317293 
99. Deng Y, Xie M, Li Q, Xu X, Ou W, Zhang Y, et al. Targeting mitochondriainflammation circuit by $\beta$-hydroxybutyrate mitigates HFpEF. Circ Res. (2021) 128:232-45. doi: 10.1161/CIRCRESAHA.120.317933

100. Tang X, Chen X, Sun X, Xu P, Zhao X, Tong Y, et al. ShortChain Enoyl-CoA hydratase mediates histone crotonylation and contributes to cardiac homeostasis. Circulation. (2021) 143:1066-9. doi: 10.1161/CIRCULATIONAHA.120.049438

101. Zhang D, Tang Z, Huang H, Zhou G, Cui C, Weng Y, et al. Metabolic regulation of gene expression by histone lactylation. Nature. (2019) 574:57580. doi: 10.1038/s41586-019-1678-1

102. Irizarry-Caro RA, Mcdaniel MM, Overcast GR, Jain VG, Troutman TD, Pasare C, et al. signaling adapter BCAP regulates inflammatory to reparatory macrophage transition by promoting histone lactylation. Proc Nat Acad Sci USA. (2020) 117:30628-38. doi: 10.1073/pnas.2009778117

103. Dai $\mathrm{X}, \mathrm{Lv} \mathrm{X}$, Thompson EK, Ostrikov KK. Histone lactylation: epigenetic mark of glycolytic switch. Trends Genet. (2021) 9:9. doi: 10.1016/j.tig.2021.09.009

104. Wang H, Jiang M, Li X, Zhao Y, Shao J, Liu Z, et al. Anti-inflammatory therapies for coronary heart disease: a systematic review and meta-analysis. Front Cardiovasc Med. (2021) 8:726341. doi: 10.3389/fcvm.2021.726341

105. Swirski FK, Nahrendorf M. Cardioimmunology: the immune system in cardiac homeostasis and disease. Nat Rev Immunol. (2018) 18:73344. doi: 10.1038/s41577-018-0065-8

106. Virani SS, Alonso A, Benjamin EJ, Bittencourt MS, Callaway CW, Carson AP, et al. Heart disease and stroke statistics-2020 update: a report from the American heart association. Circulation. (2020) 141:746. doi: 10.1161/CIR.0000000000000746

107. Stone GWM, Selker HPM, Thiele HM, Patel MRM, Udelson JEM, Ohman EMM, et al. Relationship between infarct size and outcomes following primary PCI. J Am Coll Cardiol. (2016) 67:1674-83. doi: 10.1016/j.jacc.2016.01.069

108. Yellon DM, Hausenloy DJ. Myocardial reperfusion injury. N Engl J Med. (2007) 357:1121-35. doi: 10.1056/NEJMra071667

109. Ramachandra CJA, Hernandez-Resendiz S, Crespo-Avilan GE, Lin Y, Hausenloy DJ. Mitochondria in acute myocardial infarction and cardioprotection. Ebiomedicine. (2020) 57:102884. doi: 10.1016/j.ebiom.2020.102884

110. Burkhoff DMP, Sayer GM, Doshi DM, Uriel NM. Hemodynamics of mechanical circulatory support. J Am Coll Cardiol. (2015) 66:266374. doi: $10.1016 /$ j.jacc.2015.10.017

111. Kapur NK, Paruchuri V, Pham DT, Reyelt L, Murphy B, Beale C, et al. Hemodynamic effects of left atrial or left ventricular cannulation for acute circulatory support in a bovine model of left heart injury. ASAIO J. (2015) 61:301-6. doi: 10.1097/MAT.0000000000000195

112. Curran J, Burkhoff D, Kloner RA. Beyond reperfusion: acute ventricular unloading and cardioprotection during myocardial infarction. J Cardiovasc Transl. (2019) 12:95-106. doi: 10.1007/s12265-019-9863-Z

113. Koo JH, Guan K. Interplay between YAP/TAZ and metabolism. Cell Metab. (2018) 28:196-206. doi: 10.1016/j.cmet.2018.07.010

114. Liao H, Qi Y, Ye Y, Yue P, Zhang D, Li Y. Mechanotranduction pathways in the regulation of mitochondrial homeostasis in cardiomyocytes. Front Cell Dev Biol. (2021) 8:625089. doi: 10.3389/fcell.2020.625089

115. Zhang Y, Qi Y, Li JJ, He WJ, Gao XH, Zhang Y, et al. Stretch-induced sarcoplasmic reticulum calcium leak is causatively associated with atrial fibrillation in pressure-overloaded hearts. Cardiovasc Res. (2021) 117:1091102. doi: $10.1093 / \mathrm{cvr} / \mathrm{cvaa} 163$

116. Berridge MJ. Calcium signallingremodelling and disease. Biochem Soc Trans. (2012) 40:297-309. doi: 10.1042/BST20110766

117. Boyman L, Karbowski M, Lederer WJ. Regulation of mitochondrial ATP production: $\mathrm{Ca}(2+)$ signaling and quality control. Trends Mol Med. (2020) 26:21-39. doi: 10.1016/j.molmed.2019.10.007

118. Schaeffer PJ, Wende AR, Magee CJ, Neilson JR, Leone TC, Chen F, et al. Calcineurin and Calcium/Calmodulin-dependent protein kinase activate distinct metabolic gene regulatory programs in cardiac muscle. J Biol Chem. (2004) 279:39593-603. doi: 10.1074/jbc.M403649200

119. Hajnoczky G, Robb-Gaspers LD, Seitz MB, Thomas AP. Decoding of cytosolic calcium oscillations in the mitochondria. Cell. (1995) 82:41524. doi: 10.1016/0092-8674(95)90430-1
120. Kohlhaas M, Maack C. Adverse bioenergetic consequences of na $+-\mathrm{Ca} 2+$ exchanger-mediated ca2+ influx in cardiac myocytes. Circulation. (2010) 122:2273-80. doi: 10.1161/CIRCULATIONAHA.110.968057

121. Maus M, Cuk M, Patel B, Lian J, Ouimet M, Kaufmann U, et al. Store-operated $\mathrm{ca}^{2+}$ entry controls induction of lipolysis and the transcriptional reprogramming to lipid metabolism. Cell Metab. (2017) 25:698-712. doi: 10.1016/i.cmet.2016.12.021

122. Ashrafi G, de Juan-Sanz J, Farrell RJ, Ryan TA. Molecular tuning of the axonal mitochondrial $\mathrm{Ca}^{2+}$ uniporter ensures metabolic flexibility of neurotransmission. Neuron. (2020) 105:678-87. doi: 10.1016/j.neuron.2019.11.020

123. Coste B, Mathur J, Schmidt M, Earley TJ, Ranade S, Petrus MJ, et al. Piezo1 and piezo2 are essential components of distinct mechanically activated cation channels. Science. (2010) 330:55-60. doi: 10.1126/science.1193270

124. Li J, Hou B, Tumova S, Muraki K, Bruns A, Ludlow MJ, et al. Piezo1 integration of vascular architecture with physiological force. Nature. (2014) 515:279-82. doi: 10.1038/nature13701

125. Liang J, Huang B, Yuan G, Chen Y, Liang F, Zeng H, et al. Stretch-activated channel Piezo1 is up-regulated in failure heart and cardiomyocyte stimulated by AngII. Am J Transl Res. (2017) 9:2945-55.

126. Wong $\mathrm{T}$, Juang $\mathrm{W}$, Tsai $\mathrm{C}$, Tseng $\mathrm{C}$, Lee $\mathrm{W}$, Chang $\mathrm{S}$, et al. Mechanical stretching simulates cardiac physiology and pathology through mechanosensor piezo1. J Clin Med. (2018) 7:410. doi: 10.3390/jcm7110410

127. Baratchi S, Zaldivia MTK, Wallert M, Loseff-Silver J, Al-Aryahi S, Zamani J, et al. Transcatheter aortic valve implantation represents an anti-inflammatory therapy via reduction of shear stress-induced, piezo-1-mediated monocyte activation. Circulation. (2020) 142:1092105. doi: 10.1161/CIRCULATIONAHA.120.045536

128. Duchemin A, Vignes H, Vermot J. Mechanically activated piezo channels modulate outflow tract valve development through the Yap1 and Klf2-Notch signaling axis. Elife. (2019) 8:44706. doi: 10.7554/eLife.44706

129. Yang Y, Wang D, Zhang C, Yang W, Li C, Gao Z, et al. Piezol mediates endothelial atherogenic inflammatory responses via regulation of YAP/TAZ activation. Hum Cell. (2021). doi: 10.1007/s13577-021-00600-5

130. Meng Z, Qiu Y, Lin KC, Kumar A, Placone JK, Fang C, et al. RAP2 mediates mechanoresponses of the Hippo pathway. Nature. (2018) 560:65560. doi: 10.1038/s41586-018-0444-0

131. Kashihara T, Sadoshima J. Role of YAP/TAZ in energy metabolism in the heart. J Cardiovasc Pharm. (2019) 74:48390. doi: 10.1097/FJC.0000000000000736

132. Saku K, Kakino T, Arimura T, Sunagawa G, Nishikawa T, Sakamoto T, et al. Left ventricular mechanical unloading by total support of impella in myocardial infarction reduces infarct size, preserves left ventricular function, and prevents subsequent heart failure in dogs. Circulation. (2018) 11:4397. doi: 10.1161/CIRCHEARTFAILURE.117.004397

133. Kapur NK, Paruchuri V, Urbano-Morales JA, Mackey EE, Daly GH, Qiao X, et al. Mechanically unloading the left ventricle before coronary reperfusion reduces left ventricular wall stress and myocardial infarct size. Circulation. (2013) 128:328-36. doi: 10.1161/CIRCULATIONAHA.112.000029

134. Kapur NKM, Qiao XP, Paruchuri VM, Morine KJM, Syed WB, Dow SM, et al. Mechanical pre-conditioning with acute circulatory support before reperfusion limits infarct size in acute myocardial infarction. JACC Heart Failure. (2015) 3:873-82. doi: 10.1016/j.jchf.2015.06.010

135. Esposito ML, Zhang Y, Qiao X, Reyelt L, Paruchuri V, Schnitzler GR, et al. Left ventricular unloading before reperfusion promotes functional recovery after acute myocardial infarction. J Am Coll Cardiol. (2018) 72:50114. doi: 10.1016/j.jacc.2018.05.034

136. Watanabe S, Fish K, Kovacic JC, Bikou O, Leonardson L, Nomoto K, et al. Left ventricular unloading using an impella $\mathrm{CP}$ improves coronary flow and infarct zone perfusion in ischemic heart failure. J Am Heart Assoc. (2018) 7:6462. doi: 10.1161/JAHA.117.006462

137. Briceno N, Annamalai SK, Reyelt L, Crowley P, Qiao X, Swain L, et al. Left ventricular unloading increases the coronary collateral flow index before reperfusion and reduces infarct size in a swine model of acute myocardial infarction. J Am Heart Assoc. (2019) 8:13586. doi: 10.1161/JAHA.119.0 13586

138. Kapur NK, Alkhouli MA, Demartini TJ, Faraz H, George ZH, Goodwin $\mathrm{MJ}$, et al. Unloading the left ventricle before reperfusion in patients with 
anterior ST-Segment-elevation myocardial infarction. Circulation. (2019) 139:337-46. doi: 10.1161/CIRCULATIONAHA.118.038269

139. Swain L, Reyelt L, Bhave S, Qiao X, Thomas CJ, Zweck E, et al. Transvalvular ventricular unloading before reperfusion in acute myocardial infarction. $J$ Am Coll Cardiol. (2020) 76:684-99. doi: 10.1016/j.jacc.2020.06.031

140. Lee SH, Doliba N, Osbakken M, Oz M, Mancini D. Improvement of myocardial mitochondrial function after hemodynamic support with left ventricular assist devices in patients with heart failure. J Thorac Cardiovasc Surg. (1998) 116:344-9. doi: 10.1016/S0022-5223(98)70136-9

141. Ji R, Akashi H, Drosatos K, Liao X, Jiang H, Kennel PJ, et al. Increased de novo ceramide synthesis and accumulation in failing myocardium. JCI Insight. (2017) 2:96203. doi: 10.1172/jci.insight.96203

142. Chokshi A, Drosatos K, Cheema FH, Ji R, Khawaja T, Yu S, et al. Ventricular assist device implantation corrects myocardial lipotoxicity, reverses insulin resistance, and normalizes cardiac metabolism in patients with advanced heart failure. Circulation. (2012) 125:2844-53. doi: 10.1161/CIRCULATIONAHA.111.06 0889

143. Goldenberg JR, Carley AN, Ji R, Zhang X, Fasano M, Schulze PC, et al. Preservation of acyl coenzyme a attenuates pathological and metabolic cardiac remodeling through selective lipid trafficking. Circulation. (2019) 139:2765-77. doi: 10.1161/CIRCULATIONAHA.119.039610

144. Heerdt PM, Schlame M, Jehle R, Barbone A, Burkhoff D, Blanck TJ. Disease-specific remodeling of cardiac mitochondria after a left ventricular assist device. Ann Thorac Surg. (2002) 73:1216-21. doi: 10.1016/S0003-4975(01)03621-9

145. Hui S, Ghergurovich JM, Morscher RJ, Jang C, Teng X, Lu W, et al. Glucose feeds the TCA cycle via circulating lactate. Nature. (2017) 551:1158. doi: 10.1038/nature24057

146. Diakos NA, Navankasattusas S, Abel ED, Rutter J, Mccreath L, Ferrin $\mathrm{P}$, et al. Evidence of glycolysis up-regulation and pyruvate mitochondrial oxidation mismatch during mechanical unloading of the failing human heart: Implications for cardiac reloading and conditioning. JACC Basic Transl Sci. (2016) 1:432-44. doi: 10.1016/j.jacbts.2016.06.009

147. Cluntun AA, Badolia R, Lettlova S, Parnell KM, Shankar TS, Diakos NA, et al. The pyruvate-lactate axis modulates cardiac hypertrophy and heart failure. Cell Metab. (2021) 33:629-48. doi: 10.1016/j.cmet.2020.12.003

148. Bricker DK, Taylor EB, Schell JC, Orsak T, Boutron A, Chen $\mathrm{Y}$, et al. A mitochondrial pyruvate carrier required for pyruvate uptake in yeast, drosophila, and humans. Science. (2012) 337:96-100. doi: 10.1126/science.1218099

149. Shahinian JH, Rog Zielinska EA, Schlimpert M, Mayer B, Tholen S, Kammerer B, et al. Impact of left ventricular assist device therapy on the cardiac proteome and metabolome composition in ischemic cardiomyopathy. Artif Organs. (2020) 44:257-67. doi: 10.1111/aor.13566

150. Badolia R, Ramadurai DKA, Abel ED, Ferrin P, Taleb I, Shankar TS, et al. The role of nonglycolytic glucose metabolism in myocardial recovery upon mechanical unloading and circulatory support in chronic heart failure. Circulation. (2020) 142:25974. doi: 10.1161/CIRCULATIONAHA.119.044452

151. Huang G, Huang Z, Peng Y, Wang Y, Liu W, Xue Y, et al. Metabolic processes are potential biological processes distinguishing nonischemic dilated cardiomyopathy from ischemic cardiomyopathy: a clue from serum proteomics. Pharmgenomics Pers Med. (2021) 14:1169-84. doi: 10.2147/PGPM.S323379

152. Scholz KH, Maier SKG, Maier LS, Lengenfelder B, Jacobshagen C, Jung J, et al. Impact of treatment delay on mortality in ST-segment elevation myocardial infarction (STEMI) patients presenting with and without haemodynamic instability: Results from the German prospective, multicentre FITT-STEMI trial. Eur Heart J. (2018) 39:1065-74. doi: 10.1093/eurheartj/ehy004

153. Vallabhajosyula S, Dunlay SM, Prasad A, Kashani K, Sakhuja A, Gersh BJ, et al. Acute noncardiac organ failure in acute myocardial infarction with cardiogenic shock. J Am Coll Cardiol. (2019) 73:178191. doi: 10.1016/j.jacc.2019.01.053

154. Kapur NK, Davila CD, Chweich H. Protecting the vulnerable left ventricle: the art of unloading with VA-ECMO. Circ Heart Fail. (2019) 12:e6581. doi: 10.1161/CIRCHEARTFAILURE.119.006581
155. Lüsebrink E, Orban M, Kupka D, Scherer C, Hagl C, Zimmer S, et al. Prevention and treatment of pulmonary congestion in patients undergoing venoarterial extracorporeal membrane oxygenation for cardiogenic shock. Eur Heart J. (2020) 41:3753-61. doi: 10.1093/eurheartj/eh aa547

156. Aso S, Matsui H, Fushimi K, Yasunaga H. The effect of intraaortic balloon pumping under venoarterial extracorporeal membrane oxygenation on mortality of cardiogenic patients: an analysis using a nationwide inpatient database. Crit Care Med. (2016) 44:1974-9. doi: 10.1097/CCM.0000000000001828

157. Rao P, Khalpey Z, Smith R, Burkhoff D, Kociol RD. Venoarterial extracorporeal membrane oxygenation for cardiogenic shock and cardiac arrest. Circulation. (2018) 11:4905. doi: 10.1161/CIRCHEARTFAILURE.118.004905

158. Jugdutt BI. Ischemia/infarction. Heart Fail Clin. (2012) 8:4351. doi: 10.1016/j.hfc.2011.08.006

159. Abrams D, Combes A, Brodie D. Extracorporeal membrane oxygenation in cardiopulmonary disease in adults. J Am Coll Cardiol. (2014) 63:276978. doi: 10.1016/j.jacc.2014.03.046

160. Vallabhajosyula S, Prasad A, Bell MR, Sandhu GS, Eleid MF, Dunlay $\mathrm{SM}$, et al. Extracorporeal membrane oxygenation use in acute myocardial infarction in the United States, 2000 to 2014. Circul Heart Failure. (2019) 12:e5929. doi: 10.1161/CIRCHEARTFAILURE.119.005929

161. Vallabhajosyula S, Horo JC, Antharam P, Ananthaneni S, Vallabhajosyula S, Stulak JM, et al. Concomitant Intra-Aortic balloon pump use in cardiogenic shock requiring Veno-Arterial extracorporeal membrane oxygenation. Circulation. (2018) 11:6930. doi: 10.1161/CIRCINTERVENTIONS.118.006930

162. Vallabhajosyula S, Horo JC, Antharam P, Ananthaneni S, Vallabhajosyula S, Stulak JM, et al. Venoarterial extracorporeal membrane oxygenation with concomitant impella versus venoarterial extracorporeal membrane oxygenation for cardiogenic shock. ASAIO J. (2020) 66:497-503. doi: 10.1097/MAT.0000000000001039

163. Tongers J, Sieweke J, Kühn C, Napp LC, Flierl U, Röntgen P, et al. Early escalation of mechanical circulatory support stabilizes and potentially rescues patients in refractory cardiogenic shock. Circulation. (2020) 13:5853. doi: 10.1161/CIRCHEARTFAILURE. 118.005853

164. Al-Fares AA, Randhawa VK, Englesakis M, Mcdonald MA, Nagpal AD, Estep JD, et al. Optimal strategy and timing of left ventricular venting during Veno-Arterial extracorporeal life support for adults in cardiogenic shock. Circulation. (2019) 12:6486. doi: 10.1161/CIRCHEARTFAILURE.119.006486

165. Allan CK, Newburger JW, Mcgrath E, Elder J, Psoinos C, Laussen PC, et al. The relationship between inflammatory activation and clinical outcome after infant cardiopulmonary bypass. Anesthesia Analgesia. (2010) 111:124451. doi: 10.1213/ANE.0b013e3181f333aa

166. Shew SB, Keshen TH, Jahoor F, Jaksic T. The determinants of protein catabolism in neonates on extracorporeal membrane oxygenation. J Pediatr Surg. (1999) 34:1086-90. doi: 10.1016/S0022-3468(99)90572-9

167. Priddy CMOK, Kajimoto M, Ledee DR, Bouchard B, Isern N, Olson AK, et al. Myocardial oxidative metabolism and protein synthesis during mechanical circulatory support by extracorporeal membrane oxygenation. Am J PhysiolHeart C. (2013) 304:H406-14. doi: 10.1152/ajpheart.00672.2012

168. Kajimoto M, O'Kelly Priddy CM, Ledee DR, Xu C, Isern N, Olson AK, et al. Extracorporeal membrane oxygenation promotes long chain fatty acid oxidation in the immature swine heart in vivo. J Mol Cell Cardiol. (2013) 62:144-52. doi: 10.1016/j.yjmcc.2013.05.014

169. Randle PJ, Garland PB, Hales CN, Newsholme EA. The glucose fatty-acid cycle. Its role in insulin sensitivity and the metabolic disturbances of diabetes mellitus. Lancet. (1963) 1:785-9. doi: 10.1016/S0140-6736(63)91500-9

170. Kajimoto M, O'Kelly Priddy CM, Ledee DR, Xu C, Isern N, Olson $\mathrm{AK}$, et al. Myocardial reloading after extracorporeal membrane oxygenation alters substrate metabolism while promoting protein synthesis. J Am Heart Assoc. (2013) 2:106. doi: 10.1161/JAHA.113.00 0106

171. Portman MA, Standaert TA, Ning XH. Relation of myocardial oxygen consumption and function to high energy phosphate utilization during graded hypoxia and reoxygenation in sheep 
in vivo. J Clin Invest. (1995) 95:2134-42. doi: 10.1172/JCI11 7902

172. Files MD, Kajimoto M, O’Kelly Priddy CM, Ledee DR, Xu C, Des Rosiers C, et al. Triiodothyronine facilitates weaning from extracorporeal membrane oxygenation by improved mitochondrial substrate utilization. J Am Heart Assoc. (2014) 3:680. doi: 10.1161/JAHA.113.000680

173. Des Rosiers C, Labarthe F, Lloyd SG, Chatham JC. Cardiac anaplerosis in health and disease: food for thought. Cardiovasc Res. (2011) 90:2109. doi: 10.1093/cvr/cvr055

174. Pound KM, Sorokina N, Ballal K, Berkich DA, Fasano M, Lanoue KF, et al. Substrate-enzyme competition attenuates upregulated anaplerotic flux through malic enzyme in hypertrophied rat heart and restores triacylglyceride content: Attenuating upregulated anaplerosis in hypertrophy. Circ Res. (2009) 104:805-12. doi: 10.1161/CIRCRESAHA.108.189951

175. Frerou A, Lesouhaitier M, Gregoire M, Uhel F, Gacouin A, Reizine F, et al. Venoarterial extracorporeal membrane oxygenation induces early immune alterations. Crit Care. (2021) 25:3444. doi: 10.1186/s13054-020-03444-x
Conflict of Interest: The authors declare that the research was conducted in the absence of any commercial or financial relationships that could be construed as a potential conflict of interest.

Publisher's Note: All claims expressed in this article are solely those of the authors and do not necessarily represent those of their affiliated organizations, or those of the publisher, the editors and the reviewers. Any product that may be evaluated in this article, or claim that may be made by its manufacturer, is not guaranteed or endorsed by the publisher.

Copyright (c) 2021 Jiang, Xie, Cao and Wang. This is an open-access article distributed under the terms of the Creative Commons Attribution License (CC BY). The use, distribution or reproduction in other forums is permitted, provided the original author(s) and the copyright owner(s) are credited and that the original publication in this journal is cited, in accordance with accepted academic practice. No use, distribution or reproduction is permitted which does not comply with these terms. 